\title{
Convergence of Cahn-Hilliard systems to the Stefan problem with dynamic boundary conditions
}

\author{
Takeshi Fukao \\ Department of Mathematics, Faculty of Education \\ Kyoto University of Education \\ 1 Fujinomori, Fukakusa, Fushimi-ku, Kyoto 612-8522 Japan \\ E-mail: fukao@kyokyo-u.ac.jp
}

\begin{abstract}
This paper examines the well-posedness of the Stefan problem with a dynamic boundary condition. To show the existence of the weak solution, the original problem is approximated by a limit of an equation and dynamic boundary condition of Cahn-Hilliard type. By using this Cahn-Hilliard approach, it becomes clear that the state of the mushy region of the Stefan problem is characterized by an asymptotic limit of the fourth-order system, which has a double-well structure. This fact also raises the possibility of the numerical application of the Cahn-Hilliard system to the degenerate parabolic equation, of which the Stefan problem is one.
\end{abstract}

Key words: Stefan problem, dynamic boundary condition, weak solution, CahnHilliard system.

AMS (MOS) subject classification: 80A22, 35K61, 35K25, 35D30, 47J35.

\section{Introduction}

The Stefan problem is a well-known mathematical model that describes the solid-liquid phase transition. Among many results in the literature (for example, [17, 21, 25, 27] and so on), the following enthalpy formulation of the Stefan problem with the Dirichlet-Robin type boundary condition was studied by the $L^{2}$-framework in [15]:

$$
\frac{\partial u}{\partial t}-\Delta \beta(u)=g \quad \text { in } Q:=(0, T) \times \Omega,
$$

where $0<T<+\infty$ is a finite time and $\Omega \subset \mathbb{R}^{d}, d=2$ or 3 , is a bounded domain with smooth boundary $\Gamma$. The unknown $u: \bar{Q} \rightarrow \mathbb{R}$ denotes the enthalpy and $\beta(u)$ is the temperature; $g: Q \rightarrow \mathbb{R}$ is a given heat source. In the model of the Stefan problem 
$\beta: \mathbb{R} \rightarrow \mathbb{R}$ is a piecewise linear function of the following form:

$$
\beta(r):= \begin{cases}k_{s} r & r<0, \\ 0 & 0 \leq r \leq L, \\ k_{\ell}(r-L) & r>L\end{cases}
$$

$k_{s}, k_{\ell}>0$ represet the heat conductivities on the solid and liquid regions, respectively, and $L>0$ is the latent heat constant. Consider the initial-boundary value problem for this kind of partial differential equation. The dynamic boundary condition is a sort of differential equation that represents the dynamics on the boundary $\Gamma$. As the condition for solving the partial differential equation, the time derivative is included, and this is well treated like, Dirichlet, Neumann, and Robin-type conditions for various problems. Under the dynamic boundary condition of the following form:

$$
\frac{\partial \beta(u)}{\partial t}+\partial_{\nu} \beta(u)=g_{\Gamma} \quad \text { on } \Sigma:=(0, T) \times \Gamma,
$$

the existence and uniqueness of (1.1) was studied in [1, 2] using a subdifferential approach, where the symbol $\partial_{\boldsymbol{\nu}}$ denotes the normal derivative on $\Gamma$ outward from $\Omega ; g_{\Gamma}: \Sigma \rightarrow \mathbb{R}$ is a given heat source on the boundary. For a more general setting, we can find the result in [3].

In this paper, the well-posedness of the Stefan problem with the following dynamic boundary condition is studied:

$$
\frac{\partial u}{\partial t}+\partial_{\nu} \beta(u)-\Delta_{\Gamma} \beta(u)=g_{\Gamma} \quad \text { on } \Sigma,
$$

where the symbol $\Delta_{\Gamma}$ stands for the Laplace-Beltrami operator on $\Gamma$ (see, e.g., [20, Chapter 3]). If we simultaneously consider (1.1) on the bulk $\Omega$ and (1.3) or (1.4) on the boundary $\Gamma$, then the setting of (1.4) is more natural than that of (1.3).

The main idea of the existence result is to approximate the Stefan problem from the Cahn-Hilliard system. In 2009, Goldstein, Miranville, and Schimperna studied the following Cahn-Hilliard system: For $\varepsilon>0$

$$
\begin{gathered}
\frac{\partial u}{\partial t}-\Delta \mu=0 \quad \text { in } Q \\
\mu=-\varepsilon \Delta u+\beta(u)+\varepsilon \pi(u)-f \quad \text { in } Q,
\end{gathered}
$$

with a dynamic boundary condition of the following form:

$$
\begin{gathered}
\frac{\partial u}{\partial t}+\partial_{\nu} \mu-\Delta_{\Gamma} \mu=0 \quad \text { on } \Sigma \\
\mu=\varepsilon \partial_{\nu} u-\varepsilon \Delta_{\Gamma} u+\beta_{\Gamma}(u)+\varepsilon \pi_{\Gamma}(u)-f_{\Gamma} \quad \text { on } \Sigma .
\end{gathered}
$$

The unknowns $u, \mu: \bar{Q} \rightarrow \mathbb{R}$ represent the order parameter and chemical potential, respectively. Let us recall some basic concepts. The Cahn-Hilliard system is characterized by the nonlinear terms $\beta+\pi$ and $\beta_{\Gamma}+\pi_{\Gamma}$, which are some derivatives of functions $W$ and $W_{\Gamma}$, respectively. Usually referred to as double-well potentials, for example, $W(r)=$ 
$W_{\Gamma}(r)=(1 / 4)\left(r^{2}-1\right)^{2}$, in this case $\beta(r)=\beta_{\Gamma}(r)=r^{3}$ and $\pi(r)=\pi_{\Gamma}(r)=-r$ for all $r \in \mathbb{R}$. For the details, see [6, 16]. Now, taking $\beta_{\Gamma}=\beta$ as (1.2) and letting $\varepsilon \rightarrow 0$ in (1.6), we obtain (1.1) as the limiting problem of (1.5) with $\mu=\beta(u)-f$ and some modification of $-\Delta f=g$ in $\Omega$ and $\partial_{\nu} f-\Delta_{\Gamma} f=g_{\Gamma}$ in $\Gamma$, with the setting that the trace $f_{\mid \Gamma}$ of $f$ coincides with $f_{\Gamma}$. Then, from (1.8), we can also characterize the dynamic boundary condition (1.4) by (1.7).

The existence and uniqueness of the Cahn-Hilliard system (1.5)-(1.8), as well as its asymptotic behavior, was treated by [19] in the case when $\varepsilon>0$. Following this, many related problems were treated in [8, 9]. Recently, the author extended the pioneering work of [19] to the more general case in which $\beta$ and $\beta_{\Gamma}$ are maximal monotone graphs. This included the singular case for the subdifferential $\partial I_{[-1,1]}$ of the indicator function $I_{[-1,1]}$ of the closed interval $[-1,1]$, and when $\pi$ and $\pi_{\Gamma}$ are Lipschitz continuous functions. The well-posedness of strong and weak solutions for the initial-boundary problem under appropriate assumptions was discussed in [11], where the essential idea comes from [7, 13].

The present paper proceeds as follows. In Section 2, the main theorem is stated. First, we prepare the notation used in this paper. Next, we recall a known result for some equations and dynamic boundary conditions of Cahn-Hilliard type $(\mathrm{P})_{\varepsilon}$ in Proposition 2.1. The main theorem is related to the convergence of Cahn-Hilliard systems $(\mathrm{P})_{\varepsilon}$ to the Stefan problem (P) with dynamic boundary conditions. In Section 3, we obtain a uniform estimate that is useful in proving the main theorem. To guarantee sufficient regularity of the unknowns for $(\mathrm{P})_{\varepsilon}$, we start from the approximate problem $(\mathrm{P})_{\varepsilon, \lambda}$. After obtaining all necessary estimates, we correct similar uniform estimates for $(\mathrm{P})_{\varepsilon}$. A proof of the main theorem is given in Section 4. The strategy of the proof proceeds in a standard manner. Based on the uniform estimates, we consider the limiting procedure $\varepsilon \rightarrow 0$. The main theorem is applied under a more general assumption for $\beta$, namely that it is not only a non-decreasing piecewise linear function, as in (1.2), but also some maximal monotone graph. Therefore, we apply the monotonicity argument. Finally, in Section 5, the uniqueness of $(\mathrm{P})$ is proved by showing the continuous dependence for the given data.

A detailed index of sections and subsections is as follows:

\section{Introduction}

2. Main results

\subsection{Notation}

2.2. Main theorem

2.3. Approximate solutions to the Cahn-Hilliard system $(\mathrm{P})_{\varepsilon}$

3. Uniform estimates

3.1 Uniform estimates for approximate solutions of $(\mathrm{P})_{\varepsilon, \lambda}$

3.2 Uniform estimates for approximate solutions of $(\mathrm{P})_{\varepsilon}$

4. Proof of the main theorem

5. Continuous dependence 


\section{Main results}

In this section, the main theorem is stated. First, we recall a previous result from [1] that plays an important role in this paper. Next, we present the main theorem.

\subsection{Notation}

We use the spaces $H:=L^{2}(\Omega), V:=H^{1}(\Omega), H_{\Gamma}:=L^{2}(\Gamma)$, and $V_{\Gamma}:=H^{1}(\Gamma)$ with the usual norms $|\cdot|_{H},|\cdot|_{V},|\cdot|_{H_{\Gamma}},|\cdot|_{V_{\Gamma}}$ and inner products $(\cdot, \cdot)_{H},(\cdot, \cdot)_{V},(\cdot, \cdot)_{H_{\Gamma}},(\cdot, \cdot)_{V_{\Gamma}}$, respectively. Moreover, $\boldsymbol{H}:=H \times H_{\Gamma}, \boldsymbol{V}:=\left\{\left(z, z_{\Gamma}\right) \in V \times V_{\Gamma}: z_{\Gamma}=z_{\left.\right|_{\Gamma}}\right.$ a.e. on $\left.\Gamma\right\}$, and $\boldsymbol{W}:=H^{2}(\Omega) \times H^{2}(\Gamma)$. Hereafter, we use a bold symbol $\boldsymbol{z}$ to denote the pair $\left(z, z_{\Gamma}\right)$ corresponding to the letter. Then, $\boldsymbol{H}, \boldsymbol{V}$, and $\boldsymbol{W}$ are Hilbert spaces with the inner product

$$
(\boldsymbol{u}, \boldsymbol{z})_{\boldsymbol{H}}:=(u, z)_{H}+\left(u_{\Gamma}, z_{\Gamma}\right)_{H_{\Gamma}} \text { for all } \boldsymbol{u}, \boldsymbol{z} \in \boldsymbol{H},
$$

and the related norm is analogously defined as one of $\boldsymbol{V}$ or $\boldsymbol{W}$. Note that, if $\boldsymbol{z} \in \boldsymbol{V}$, then $z_{\Gamma}$ is exactly the trace $z_{\left.\right|_{\Gamma}}$ of $z$ on $\Gamma$, whereas if $\boldsymbol{z}$ is just in $\boldsymbol{H}$, then $z \in H$ and $z_{\Gamma} \in H_{\Gamma}$ are independent. Define $m: \boldsymbol{H} \rightarrow \mathbb{R}$ by

$$
m(\boldsymbol{z}):=\frac{1}{|\Omega|+|\Gamma|}\left\{\int_{\Omega} z d x+\int_{\Gamma} z_{\Gamma} d \Gamma\right\} \quad \text { for all } \boldsymbol{z} \in \boldsymbol{H}
$$

where $|\Omega|:=\int_{\Omega} 1 d x$ and $|\Gamma|:=\int_{\Gamma} 1 d \Gamma$. The symbol $\boldsymbol{V}^{*}$ denotes the dual space of $\boldsymbol{V}$, and the pair $\langle\cdot, \cdot\rangle_{\boldsymbol{V}^{*}, \boldsymbol{V}}$ denotes the duality pairing between $\boldsymbol{V}^{*}$ and $\boldsymbol{V}$. Moreover, we define the bilinear form $a(\cdot, \cdot): \boldsymbol{V} \times \boldsymbol{V} \rightarrow \mathbb{R}$ by

$$
a(\boldsymbol{u}, \boldsymbol{z}):=\int_{\Omega} \nabla u \cdot \nabla z d x+\int_{\Gamma} \nabla_{\Gamma} u_{\Gamma} \cdot \nabla_{\Gamma} z_{\Gamma} d \Gamma \quad \text { for all } \boldsymbol{u}, \boldsymbol{z} \in \boldsymbol{V}
$$

where $\nabla_{\Gamma}$ denotes the surface gradient on $\Gamma$ (see, e.g., [20, Chapter 3]). We also introduce the subspace of $\boldsymbol{H}$ as $\boldsymbol{H}_{0}:=\{\boldsymbol{z} \in \boldsymbol{H}: m(\boldsymbol{z})=0\}$ and $\boldsymbol{V}_{0}:=\boldsymbol{V} \cap \boldsymbol{H}_{0}$ with their norms $|\boldsymbol{z}|_{\boldsymbol{H}_{0}}:=|\boldsymbol{z}|_{\boldsymbol{H}}$ for all $\boldsymbol{z} \in \boldsymbol{H}_{0}$ and $|\boldsymbol{z}|_{\boldsymbol{V}_{0}}:=a(\boldsymbol{z}, \boldsymbol{z})^{1 / 2}$ for all $\boldsymbol{z} \in \boldsymbol{V}_{0}$. Then, we can define the duality mapping $\boldsymbol{F}: \boldsymbol{V}_{0} \rightarrow \boldsymbol{V}_{0}^{*}$ by

$$
\langle\boldsymbol{F} \boldsymbol{z}, \tilde{\boldsymbol{z}}\rangle_{\boldsymbol{V}_{0}^{*}, \boldsymbol{V}_{0}}:=a(\boldsymbol{z}, \tilde{\boldsymbol{z}}) \text { for all } \boldsymbol{z}, \tilde{\boldsymbol{z}} \in \boldsymbol{V}_{0}
$$

Using this, we can define the inner product in $\boldsymbol{V}_{0}^{*}$ by

$$
\left(\boldsymbol{z}_{1}^{*}, \boldsymbol{z}_{2}^{*}\right)_{\boldsymbol{V}_{0}^{*}}:=\left\langle\boldsymbol{z}_{1}^{*}, \boldsymbol{F}^{-1} \boldsymbol{z}_{2}^{*}\right\rangle_{\boldsymbol{V}_{0}^{*}, \boldsymbol{V}_{0}} \text { for all } \boldsymbol{z}_{1}^{*}, \boldsymbol{z}_{2}^{*} \in \boldsymbol{V}_{0}^{*}
$$

Then, we obtain the dense and compact embeddings $\boldsymbol{V}_{0} \hookrightarrow \hookrightarrow \boldsymbol{H}_{0} \hookrightarrow \hookrightarrow \boldsymbol{V}_{0}^{*}$; see [1] for details of this setting. These are essentially the same as in previous studies [10, 23, 24].

\subsection{Main theorem}

In this subsection, we define the weak solution for the Stefan problem with a dynamic boundary condition. Then, we give the main theorem. 
First, we present the target problem (P) of this paper, namely the Stefan problem with the dynamic boundary condition:

$$
\begin{gathered}
\frac{\partial u}{\partial t}-\Delta \beta(u)=g \quad \text { a.e. in } Q, \\
\beta\left(u_{\Gamma}\right)=\beta(u)_{\left.\right|_{\Gamma}}, \quad \frac{\partial u_{\Gamma}}{\partial t}+\partial_{\nu} \beta(u)-\Delta_{\Gamma} \beta\left(u_{\Gamma}\right)=g_{\Gamma} \quad \text { a.e. on } \Sigma, \\
u(0)=u_{0} \quad \text { a.e. in } \Omega, \quad u_{\Gamma}(0)=u_{0 \Gamma} \quad \text { a.e. on } \Gamma,
\end{gathered}
$$

where the prototype Stefan problem is formulated by the setting (1.2). In this paper, the target problem $(\mathrm{P})$ will be formulated in a more general setting; see Definition 2.1.

Remark 1. The dynamic boundary condition is arranged from three previous results [1, 2, 3] regarding not only the Laplace-Beltrami operator, but also the time derivative. Actually, they treated $\partial \beta\left(u_{\Gamma}\right) / \partial t$. In the case of (1.2), the first condition (2.4) implies that $u_{\Gamma}$ is not necessarily equal to the trace $u_{\left.\right|_{\Gamma}}$ of $u$. More precisely, we will obtain $u_{\Gamma}=u_{\left.\right|_{\Gamma}}$ except in the mushy region $\{x \in \Omega: 0 \leq u(x) \leq L\}$ of the Stefan problem. This is because we can only expect $\beta(u(t)) \in V$, but $u(t) \notin V$ (weak solution).

Throughout this paper, we assume that

(A1) $\beta$ is a maximal monotone graph in $\mathbb{R} \times \mathbb{R}$, and is a subdifferential $\beta=\partial \widehat{\beta}$ of some proper, lower semicontinuous, and convex function $\widehat{\beta}: \mathbb{R} \rightarrow[0,+\infty]$ satisfying $\widehat{\beta}(0)=0$ with some effective domain $D(\beta)$. This implies $\beta(0)=0$. Moreover, there exist two constants $c_{1}, c_{2}>0$ such that

$$
\widehat{\beta}(r) \geq c_{1}|r|^{2}-c_{2} \quad \text { for all } r \in \mathbb{R}
$$

(A2) $\pi: D(\pi)=\mathbb{R} \rightarrow \mathbb{R}$ is Lipschitz continuous. Moreover,

$$
\left|\pi^{\prime}(r)\right| \leq 1 \quad \text { for a.a. } r \in \mathbb{R}
$$

(A3) $\boldsymbol{g} \in L^{2}\left(0, T ; \boldsymbol{H}_{0}\right)$;

(A4) $\boldsymbol{u}_{0}:=\left(u_{0}, u_{0 \Gamma}\right) \in \boldsymbol{V}$ with $m_{0} \in \operatorname{int} D(\beta)$, and the compatibility conditions $\widehat{\beta}\left(u_{0}\right) \in$ $L^{1}(\Omega), \widehat{\beta}\left(u_{0 \Gamma}\right) \in L^{1}(\Gamma)$ hold.

For simplicity, we have assumed that the derivative of $\pi$ is bounded by 1 in (2.6). It is sufficient to assume that $\pi \in W^{1, \infty}(\mathbb{R})$. We now define a weak solution.

Definition 2.1. The pair $(\boldsymbol{u}, \boldsymbol{\xi})$ of functions $\boldsymbol{u} \in H^{1}\left(0, T ; \boldsymbol{V}^{*}\right) \cap L^{2}(0, T ; \boldsymbol{H})$ and $\boldsymbol{\xi} \in$ $L^{2}(0, T ; \boldsymbol{V})$ is called the weak solution of $(\mathrm{P})$ if

$$
\xi \in \beta(u) \quad \text { a.e. in } Q, \quad \xi_{\Gamma} \in \beta\left(u_{\Gamma}\right), \quad \xi_{\Gamma}=\xi_{\left.\right|_{\Gamma}} \quad \text { a.e. on } \Sigma,
$$


and they satisfy

$$
\begin{aligned}
\left\langle u^{\prime}(t), z\right\rangle_{V^{*}, V} & +\left\langle u_{\Gamma}^{\prime}(t), z_{\Gamma}\right\rangle_{V_{\Gamma}^{*}, V_{\Gamma}}+\int_{\Omega} \nabla \xi(t) \cdot \nabla z d x+\int_{\Gamma} \nabla_{\Gamma} \xi_{\Gamma}(t) \cdot \nabla_{\Gamma} z_{\Gamma} d \Gamma \\
& =\int_{\Omega} g(t) z d x+\int_{\Gamma} g_{\Gamma}(t) z_{\Gamma} d \Gamma \quad \text { for all } \boldsymbol{z}=\left(z, z_{\Gamma}\right) \in \boldsymbol{V}
\end{aligned}
$$

for a.a. $t \in(0, T)$ with $u(0)=u_{0}$ a.e. in $\Omega$ and $u_{\Gamma}(0)=u_{0 \Gamma}$ a.e. on $\Gamma$.

Our main theorem is now stated.

Theorem 2.1. Assume (A1)-(A4) hold. Then, there exists at least one weak solution $(\boldsymbol{u}, \boldsymbol{\xi})$ of $(\mathrm{P})$.

Moreover, we obtain the following continuous dependence for the given data:

Theorem 2.2. Assume (A1)-(A4). For $i=1,2$, let $\left(\boldsymbol{u}^{(i)}, \boldsymbol{\xi}^{(i)}\right)$ be a weak solution of (P) corresponding to the data $\boldsymbol{g}^{(i)}$ and $\boldsymbol{u}_{0}^{(i)}$, in which we assume that $m\left(\boldsymbol{u}_{0}^{(1)}\right)=m\left(\boldsymbol{u}_{0}^{(2)}\right)$. Then, there exists a positive constant $C$ that depends only on $T$ such that

$$
\left|\boldsymbol{u}^{(1)}(t)-\boldsymbol{u}^{(2)}(t)\right|_{\boldsymbol{V}_{0}^{*}}^{2} \leq C\left\{\left|\boldsymbol{u}_{0}^{(1)}-\boldsymbol{u}_{0}^{(2)}\right|_{\boldsymbol{V}_{0}^{*}}^{2}+\int_{0}^{T}\left|\boldsymbol{g}^{(1)}(s)-\boldsymbol{g}^{(2)}(s)\right|_{\boldsymbol{H}_{0}}^{2} d s\right\}
$$

for all $t \in[0, T]$. Moreover, if $\beta$ is Lipschitz continuous, then

$$
\begin{aligned}
& \int_{0}^{t}\left|\boldsymbol{\xi}^{(1)}(s)-\boldsymbol{\xi}^{(2)}(s)\right|_{\boldsymbol{H}}^{2} d s \\
& \leq \frac{c_{\beta}}{2} C(1+T)\left\{\left|\boldsymbol{u}_{0}^{(1)}-\boldsymbol{u}_{0}^{(2)}\right|_{\boldsymbol{V}_{0}^{*}}^{2}+\int_{0}^{T}\left|\boldsymbol{g}^{(1)}(s)-\boldsymbol{g}^{(2)}(s)\right|_{\boldsymbol{H}_{0}}^{2} d s\right\}
\end{aligned}
$$

for all $t \in[0, T]$, where $c_{\beta}>0$ is the Lipschitz constant of $\beta$.

Note that the assumption $m\left(\boldsymbol{u}_{0}^{(1)}\right)=m\left(\boldsymbol{u}_{0}^{(2)}\right)$ gives us that $\boldsymbol{u}^{(1)}(t)-\boldsymbol{u}^{(2)}(t) \in \boldsymbol{V}_{0}^{*}$ for all $t \in[0, T]$. This theorem implies the uniqueness of the weak solution of $(\mathrm{P})$.

\subsection{Approximate solutions to the Cahn-Hilliard system $(\mathrm{P})_{\varepsilon}$}

In this subsection, we state the approximate problem for $(\mathrm{P})$. For this aim, we recall a previous result [11] for the equation and dynamic boundary condition of the CahnHilliard type $(\mathrm{P})_{\varepsilon}$. This can be written as the following initial-boundary value problem 
(2.10) $-(2.14):$ For $\varepsilon>0$,

$$
\begin{gathered}
\frac{\partial u}{\partial t}-\Delta \mu=0 \quad \text { a.e. in } Q, \\
\xi \in \beta(u), \quad \mu=-\varepsilon \Delta u+\xi+\varepsilon \pi(u)-f \quad \text { a.e. in } Q, \\
u_{\Gamma}=u_{\left.\right|_{\Gamma}}, \quad \mu_{\Gamma}=\mu_{\Gamma}, \quad \frac{\partial u_{\Gamma}}{\partial t}+\partial_{\nu} \mu-\Delta_{\Gamma} \mu_{\Gamma}=0 \quad \text { a.e. on } \Sigma, \\
\xi_{\Gamma} \in \beta\left(u_{\Gamma}\right), \quad \mu_{\Gamma}=\varepsilon \partial_{\nu} u-\varepsilon \Delta_{\Gamma} u_{\Gamma}+\xi_{\Gamma}+\varepsilon \pi\left(u_{\Gamma}\right)-f_{\Gamma} \quad \text { a.e. on } \Sigma, \\
u(0)=u_{0} \quad \text { a.e. in } \Omega, \quad u_{\Gamma}(0)=u_{0 \Gamma} \quad \text { a.e. on } \Gamma,
\end{gathered}
$$

where $f: Q \rightarrow \mathbb{R}, f_{\Gamma}: \Sigma \rightarrow \mathbb{R}, u_{0}: \Omega \rightarrow \mathbb{R}$, and $u_{0 \Gamma}: \Gamma \rightarrow \mathbb{R}$ are given data. In particular, $f$ and $f_{\Gamma}$ are constructed by $g$ and $g_{\Gamma}$ at a later point. In the main theorem, we treated the general setting (A1) and (A2) of $\beta$ for the Stefan problem with some suitable $\pi$. In the setting of (1.2), one example of $\pi: \mathbb{R} \rightarrow \mathbb{R}$ is a piecewise linear function of the following form:

$$
\beta(r):=\left\{\begin{array}{ll}
k_{s} r & \text { if } r<0, \\
0 & \text { if } 0 \leq r \leq L, \\
k_{\ell}(r-L) & \text { if } r>L,
\end{array} \pi(r):= \begin{cases}\frac{L}{2} & \text { if } r<0, \\
\frac{L}{2}-r & \text { if } 0 \leq r \leq L, \\
-\frac{L}{2} & \text { if } r>L,\end{cases}\right.
$$

of course, in this case $\xi=\beta(u)$ and $\xi_{\Gamma}=\beta\left(u_{\Gamma}\right)$. Therefore, (A1) and (A2) hold; actually $\widehat{\beta}$ is obtained by

$$
\widehat{\beta}(r):= \begin{cases}\frac{k_{s}}{2} r^{2} & \text { if } r<0 \\ 0 & \text { if } 0 \leq r \leq L \\ \frac{k_{\ell}}{2}(r-L)^{2} & \text { if } r>L\end{cases}
$$

so that $\beta=\partial \widehat{\beta}$, and this quadratic function $\widehat{\beta}$ satisfies (2.5). Because of this value of $\varepsilon \pi$, we can realize the double-well structure of the potential $W=\widehat{\beta}+\varepsilon \widehat{\pi}$ as the sum of primitives of $\beta$ and $\varepsilon \pi$. Therefore, $(\mathrm{P})_{\varepsilon}$ with the prototype setting (2.15) has the exact structure of the Cahn-Hilliard system.

Remark 2. Viewed in terms of the Stefan problem, $\pi$ is an artificial term. Therefore, we can prove the existence of the weak solution of the Stefan problem even if $\pi \equiv 0$. However, in this case, we no longer find the structure of the Cahn-Hilliard system (more precisely, the double-well structure) at the level of $(\mathrm{P})_{\varepsilon}$. If we construct the relationship between some equation of Cahn-Hilliard type and the original Stefan problem, we must choose a suitable $\pi$ depending on $\beta$, which breaks the monotonicity of $\beta$. This treatment is completely independent of the choice of boundary condition. We will focus on the convergence from the Cahn-Hilliard system to the nonlinear diffusion equation under the Neumann boundary condition in a forthcoming paper [12]. 
Here, we know that for each $\boldsymbol{g}:=\left(g, g_{\Gamma}\right) \in L^{2}\left(0, T ; \boldsymbol{H}_{0}\right)$, there exists $\boldsymbol{f} \in L^{2}\left(0, T ; \boldsymbol{V}_{0}\right)$ such that

$$
\int_{\Omega} \nabla f \cdot \nabla z d x+\int_{\Gamma} \nabla_{\Gamma} f_{\Gamma} \cdot \nabla_{\Gamma} z_{\Gamma} d \Gamma=\int_{\Omega} g z d x+\int_{\Gamma} g_{\Gamma} z_{\Gamma} d \Gamma \quad \text { for all } \boldsymbol{z} \in \boldsymbol{V}_{0}
$$

a.e. in $(0, T)$ (see (2.23) $(2.24)$, also [11, Lemma C]). To state the previous known results, we use the change of variable $\boldsymbol{v}:=\boldsymbol{u}-m_{0} \mathbf{1}$, where $m_{0}:=m\left(\boldsymbol{u}_{0}\right)$ and $\mathbf{1}:=(1,1)$, the pair of constants. We also use $\boldsymbol{v}_{0}:=\boldsymbol{u}_{0}-m_{0} \mathbf{1}$. We set $\boldsymbol{\beta}(\boldsymbol{z}):=\left(\beta(z), \beta\left(z_{\Gamma}\right)\right.$ ), $\boldsymbol{\pi}(\boldsymbol{z}):=\left(\pi(z), \pi\left(z_{\Gamma}\right)\right)$ for all $\boldsymbol{z} \in \boldsymbol{H}$. Under assumptions (A1)-(A4), [11, Theorem 2.2] leads to the following proposition of the existence and uniqueness for the equation and dynamic boundary condition of Cahn-Hilliard type $(\mathrm{P})_{\varepsilon}$ with respect to our $\beta+\varepsilon \pi$.

Proposition 2.1. Under assumptions (A1)-(A4), there exists a triplet $\left(\boldsymbol{v}_{\varepsilon}, \boldsymbol{\mu}_{\varepsilon}, \boldsymbol{\xi}_{\varepsilon}\right)$ of

$$
\begin{gathered}
\boldsymbol{v}_{\varepsilon} \in H^{1}\left(0, T ; \boldsymbol{V}_{0}^{*}\right) \cap L^{\infty}\left(0, T ; \boldsymbol{V}_{0}\right) \cap L^{2}(0, T ; \boldsymbol{W}), \\
\boldsymbol{\mu}_{\varepsilon} \in L^{2}(0, T ; \boldsymbol{V}), \quad \boldsymbol{\xi}_{\varepsilon} \in L^{2}(0, T ; \boldsymbol{H}),
\end{gathered}
$$

that satisfy

$$
\begin{gathered}
\left\langle\boldsymbol{v}_{\varepsilon}^{\prime}(t), \boldsymbol{z}\right\rangle_{\boldsymbol{V}_{0}^{*}, \boldsymbol{V}_{0}}+a\left(\boldsymbol{\mu}_{\varepsilon}(t), \boldsymbol{z}\right)=0 \quad \text { for all } \boldsymbol{z} \in \boldsymbol{V}_{0}, \\
\left(\boldsymbol{\mu}_{\varepsilon}(t), \boldsymbol{z}\right)_{\boldsymbol{H}}=\varepsilon a\left(\boldsymbol{v}_{\varepsilon}(t), \boldsymbol{z}\right)+\left(\boldsymbol{\xi}_{\varepsilon}(t)+\varepsilon \boldsymbol{\pi}\left(\boldsymbol{v}_{\varepsilon}(t)+m_{0} \mathbf{1}\right)-\boldsymbol{f}(t), \boldsymbol{z}\right)_{\boldsymbol{H}} \\
\text { for all } \boldsymbol{z} \in \boldsymbol{V},
\end{gathered}
$$

for a.a. $t \in(0, T)$, with

$$
\xi_{\varepsilon} \in \beta\left(v_{\varepsilon}+m_{0}\right) \quad \text { a.e. in } Q, \quad \xi_{\Gamma, \varepsilon} \in \beta\left(v_{\Gamma, \varepsilon}+m_{0}\right) \quad \text { a.e. on } \Sigma \text {. }
$$

Moreover, $\boldsymbol{v}_{\varepsilon}(0)=\boldsymbol{v}_{0}$ in $\boldsymbol{H}_{0}$ holds.

We call the solution obtained by this proposition a kind of weak solution for the problem in (2.10)-(2.14). This proposition is a direct consequence of [11, Theorem 2.2]. Indeed, we assumed (A1)-(A4), and then, from the construction (2.16) of $\boldsymbol{f}$, we see that $\boldsymbol{f} \in L^{2}\left(0, T ; \boldsymbol{V}_{0}\right)$. Thus, all of the conditions needed to apply [11, Theorem 2.2] can be corrected.

To obtain uniform estimates with respect to $\varepsilon \in(0,1]$, we must consider the approximate problem for $(\mathrm{P})_{\varepsilon}$, which is the same strategy used to prove Proposition 2.1. Therefore, we give only a sketch of the proof here. For each $\lambda \in(0,1]$, consider the approximate problem $(\mathrm{P})_{\varepsilon, \lambda}$

$$
\begin{aligned}
& \left\langle\boldsymbol{v}_{\varepsilon, \lambda}^{\prime}(t), \boldsymbol{z}\right\rangle_{\boldsymbol{V}_{0}^{*}, \boldsymbol{V}_{0}}+a\left(\boldsymbol{\mu}_{\varepsilon, \lambda}(t), \boldsymbol{z}\right)=0 \quad \text { for all } \boldsymbol{z} \in \boldsymbol{V}_{0}, \\
\left(\boldsymbol{\mu}_{\varepsilon, \lambda}(t), \boldsymbol{z}\right)_{\boldsymbol{H}}= & \lambda\left(\boldsymbol{v}_{\varepsilon, \lambda}^{\prime}(t), \boldsymbol{z}\right)_{\boldsymbol{H}}+\varepsilon a\left(\boldsymbol{v}_{\varepsilon, \lambda}(t), \boldsymbol{z}\right) \\
& +\left(\boldsymbol{\beta}_{\lambda}\left(\boldsymbol{v}_{\varepsilon, \lambda}(t)+m_{0} \mathbf{1}\right)+\varepsilon \boldsymbol{\pi}\left(\boldsymbol{v}_{\varepsilon, \lambda}(t)+m_{0} \mathbf{1}\right)-\boldsymbol{f}(t), \boldsymbol{z}\right)_{\boldsymbol{H}} \quad \text { for all } \boldsymbol{z} \in \boldsymbol{V},
\end{aligned}
$$


with $\boldsymbol{v}_{\varepsilon, \lambda}(0)=\boldsymbol{v}_{0}$ in $\boldsymbol{H}_{0}$, where $\boldsymbol{\beta}_{\lambda}(\boldsymbol{z}):=\left(\beta_{\lambda}(z), \beta_{\lambda}\left(z_{\Gamma}\right)\right)$ for all $\boldsymbol{z} \in \boldsymbol{H}$ and $\beta_{\lambda}$ is the Yosida approximation (see, e.g., [4, 5, 22]). Namely, $\beta_{\lambda}: \mathbb{R} \rightarrow \mathbb{R}$ along with the associated resolvent operator $J_{\lambda}: \mathbb{R} \rightarrow \mathbb{R}$ are defined by

$$
\beta_{\lambda}(r):=\frac{1}{\lambda}\left(r-J_{\lambda}(r)\right):=\frac{1}{\lambda}\left(r-(I+\lambda \beta)^{-1}(r)\right) \text { for all } r \in \mathbb{R} .
$$

Moreover, the related Moreau-Yosida regularization $\widehat{\beta}_{\lambda}$ of $\widehat{\beta}: \mathbb{R} \rightarrow \mathbb{R}$ fulfills

$$
\widehat{\beta}_{\lambda}(r):=\inf _{l \in \mathbb{R}}\left\{\frac{1}{2 \lambda}|r-l|^{2}+\widehat{\beta}(l)\right\}=\frac{1}{2 \lambda}\left|r-J_{\lambda}(r)\right|^{2}+\widehat{\beta}\left(J_{\lambda}(r)\right)=\int_{0}^{r} \beta_{\lambda}(l) d l
$$

for all $r \in \mathbb{R}$. We know the basic property

$$
0 \leq \widehat{\beta}_{\lambda}(r) \leq \widehat{\beta}(r) \text { for all } r \in \mathbb{R} .
$$

The problem $(\mathrm{P})_{\varepsilon, \lambda}$ can be solved (see, e.g., [10, 11, 14, 23, 24]); more precisely, there exist $\boldsymbol{v}_{\varepsilon, \lambda} \in H^{1}\left(0, T ; \boldsymbol{H}_{0}\right) \cap L^{\infty}\left(0, T ; \boldsymbol{V}_{0}\right) \cap L^{2}(0, T ; \boldsymbol{W})$ and $\boldsymbol{\mu}_{\varepsilon, \lambda} \in L^{2}(0, T ; \boldsymbol{V})$ that satisfy

$$
\begin{aligned}
\lambda \boldsymbol{v}_{\varepsilon, \lambda}^{\prime}(t) & +\boldsymbol{F}^{-1} \boldsymbol{v}_{\varepsilon, \lambda}^{\prime}(t)+\varepsilon \partial \varphi\left(\boldsymbol{v}_{\varepsilon, \lambda}(t)\right) \\
& =\boldsymbol{P}\left(-\boldsymbol{\beta}_{\lambda}\left(\boldsymbol{v}_{\varepsilon, \lambda}(t)+m_{0} \mathbf{1}\right)-\varepsilon \boldsymbol{\pi}\left(\boldsymbol{v}_{\varepsilon, \lambda}(t)+m_{0} \mathbf{1}\right)+\boldsymbol{f}(t)\right) \quad \text { in } \boldsymbol{H}_{0}
\end{aligned}
$$

for a.a. $t \in(0, T)$ with $\boldsymbol{v}_{\varepsilon, \lambda}(0)=\boldsymbol{v}_{0}$ in $\boldsymbol{H}_{0}$, where $\varphi: \boldsymbol{H}_{0} \rightarrow[0,+\infty]$ is a proper, lower semicontinuous, and convex functional

$$
\varphi(\boldsymbol{z}):=\left\{\begin{array}{l}
\frac{1}{2} \int_{\Omega}|\nabla z|^{2} d x+\frac{1}{2} \int_{\Gamma}\left|\nabla_{\Gamma} z_{\Gamma}\right|^{2} d \Gamma \quad \text { if } \boldsymbol{z} \in \boldsymbol{V}_{0}, \\
+\infty \quad \text { otherwise. }
\end{array}\right.
$$

Here, the subdifferential $\partial \varphi$ on $\boldsymbol{H}_{0}$ is characterized by $\partial \varphi(\boldsymbol{z})=\left(-\Delta z, \partial_{\boldsymbol{\nu}} z-\Delta_{\Gamma} z_{\Gamma}\right)$ with $\boldsymbol{z} \in D(\partial \varphi)=\boldsymbol{W} \cap \boldsymbol{V}_{0}$ (see, e.g., [11, Lemma C]). We also note that

$$
2 \varphi(\boldsymbol{z})=a(\boldsymbol{z}, \boldsymbol{z})=\langle\boldsymbol{F} \boldsymbol{z}, \boldsymbol{z}\rangle_{\boldsymbol{V}_{0}^{*}, \boldsymbol{V}_{0}}=|\boldsymbol{z}|_{\boldsymbol{V}_{0}}^{2} \text { for all } \boldsymbol{z} \in \boldsymbol{V}_{0} .
$$

Moreover, $\boldsymbol{P}: \boldsymbol{H} \rightarrow \boldsymbol{H}_{0}$ is a projection defined by $\boldsymbol{P} \boldsymbol{z}:=\boldsymbol{z}-m(\boldsymbol{z}) \mathbf{1}$ for all $\boldsymbol{z} \in \boldsymbol{H}$, and it satisfies

$$
\begin{array}{ll}
\left(\boldsymbol{z}_{0}, \boldsymbol{P} \tilde{\boldsymbol{z}}\right)_{\boldsymbol{H}_{0}}=\left(\boldsymbol{z}_{0}, \tilde{\boldsymbol{z}}\right)_{\boldsymbol{H}} & \text { for all } \boldsymbol{z}_{0} \in \boldsymbol{H}_{0} \text { and } \tilde{\boldsymbol{z}} \in \boldsymbol{H} \\
|\boldsymbol{P} \boldsymbol{z}|_{\boldsymbol{V}_{0}}=|\boldsymbol{z}|_{\boldsymbol{V}_{0}} \leq|\boldsymbol{z}|_{\boldsymbol{V}} & \text { for all } \boldsymbol{z} \in \boldsymbol{V}
\end{array}
$$

The standard strategy obtains a priori estimates with respect to $\lambda \in(0,1]$ and considers the limiting procedure $\lambda \rightarrow 0$.

Remark 3. Let $\boldsymbol{u}_{\varepsilon}:=\boldsymbol{v}_{\varepsilon}+m_{0}$ 1. Then, [11, Remark 2] means that (2.17) implies

$$
\left\langle u_{\varepsilon}^{\prime}(t), z\right\rangle_{V^{*}, V}+\left\langle u_{\Gamma, \varepsilon}^{\prime}(t), z_{\Gamma}\right\rangle_{V_{\Gamma}^{*}, V_{\Gamma}}+\int_{\Omega} \nabla \mu_{\varepsilon}(t) \cdot \nabla z d x+\int_{\Gamma} \nabla_{\Gamma} \mu_{\Gamma, \varepsilon}(t) \cdot \nabla_{\Gamma} z_{\Gamma} d \Gamma=0
$$

for all $\boldsymbol{z} \in \boldsymbol{V}$. Moreover, thanks to the regularity $\boldsymbol{v}_{\varepsilon} \in L^{2}(0, T ; \boldsymbol{W})$, we see that (2.18) implies

$$
\begin{gathered}
\xi_{\varepsilon} \in \beta\left(u_{\varepsilon}\right), \quad \mu_{\varepsilon}=-\varepsilon \Delta u_{\varepsilon}+\xi_{\varepsilon}+\varepsilon \pi\left(u_{\varepsilon}\right)-f \quad \text { a.e. in } Q, \\
\xi_{\Gamma, \varepsilon} \in \beta\left(u_{\Gamma, \varepsilon}\right), \quad \mu_{\Gamma, \varepsilon}=\varepsilon \partial_{\nu} u_{\varepsilon}-\varepsilon \Delta_{\Gamma} u_{\Gamma, \varepsilon}+\xi_{\Gamma, \varepsilon}+\varepsilon \pi_{\Gamma}\left(u_{\Gamma, \varepsilon}\right)-f_{\Gamma} \quad \text { a.e. on } \Sigma .
\end{gathered}
$$




\section{Uniform estimates}

In this section, we obtain the uniform estimates needed to prove the main theorem.

\subsection{Uniform estimates for approximate solutions of $(P)_{\varepsilon, \lambda}$}

To prove the main theorem, we will use the uniform estimates that are independent of $\varepsilon$ for the solutions of $(\mathrm{P})_{\varepsilon}$. For this, we must start from the approximate problem $(\mathrm{P})_{\varepsilon, \lambda}$. In this subsection, we obtain uniform estimates for the approximate solutions of $(\mathrm{P})_{\varepsilon, \lambda}$. We recall the change of variable $\boldsymbol{u}_{\varepsilon, \lambda}:=\boldsymbol{v}_{\varepsilon, \lambda}+m_{0} \mathbf{1}$.

Lemma 3.1. There exist positive constants $M_{1}$ and $M_{2}$, independent of $\varepsilon \in(0,1 / 4]$ and $\lambda \in(0,1]$, such that

$$
\begin{gathered}
\lambda\left|\boldsymbol{v}_{\varepsilon, \lambda}(t)\right|_{\boldsymbol{H}_{0}}^{2}+\left|\boldsymbol{v}_{\varepsilon, \lambda}(t)\right|_{\boldsymbol{V}_{0}^{*}}^{2} \leq M_{1}, \\
\frac{\varepsilon}{2} \int_{0}^{t}\left|\boldsymbol{v}_{\varepsilon, \lambda}(s)\right|_{\boldsymbol{V}_{0}}^{2} d s+2 \int_{0}^{t}\left|\widehat{\beta}_{\lambda}\left(u_{\varepsilon, \lambda}(s)\right)\right|_{L^{1}(\Omega)} d s+2 \int_{0}^{t}\left|\widehat{\beta}_{\lambda}\left(u_{\Gamma, \varepsilon, \lambda}(s)\right)\right|_{L^{1}(\Gamma)} d s \leq M_{2}
\end{gathered}
$$

for all $t \in[0, T]$.

Proof. We test (2.22) at time $s \in(0, T)$ by $\boldsymbol{v}_{\varepsilon, \lambda}(s) \in \boldsymbol{V}_{0}$ which is considered in problem $(\mathrm{P})_{\varepsilon, \lambda}$. Then, using (2.3), we have

$$
\begin{aligned}
& \lambda\left(\boldsymbol{v}_{\varepsilon, \lambda}^{\prime}(s), \boldsymbol{v}_{\varepsilon, \lambda}(s)\right)_{\boldsymbol{H}_{0}}+\left(\boldsymbol{v}_{\varepsilon, \lambda}^{\prime}(s), \boldsymbol{v}_{\varepsilon, \lambda}(s)\right)_{\boldsymbol{V}_{0}^{*}}+\varepsilon\left(\partial \varphi\left(\boldsymbol{v}_{\varepsilon, \lambda}(s)\right), \boldsymbol{v}_{\varepsilon, \lambda}(s)\right)_{\boldsymbol{H}_{0}} \\
& \quad+\left(\boldsymbol{P} \boldsymbol{\beta}_{\lambda}\left(\boldsymbol{v}_{\varepsilon, \lambda}(s)+m_{0} \mathbf{1}\right), \boldsymbol{v}_{\varepsilon, \lambda}(s)\right)_{\boldsymbol{H}_{0}}=\left(\boldsymbol{f}(s)-\varepsilon \boldsymbol{P} \boldsymbol{\pi}\left(\boldsymbol{v}_{\varepsilon, \lambda}(s)\right), \boldsymbol{v}_{\varepsilon, \lambda}(s)\right)_{\boldsymbol{H}_{0}}
\end{aligned}
$$

for a.a. $s \in(0, T)$. Now, from the definition of the subdifferential, we have

$$
\begin{aligned}
& \left(\boldsymbol{P} \boldsymbol{\beta}_{\lambda}\left(\boldsymbol{v}_{\varepsilon, \lambda}(s)+m_{0} \mathbf{1}\right), \boldsymbol{v}_{\varepsilon, \lambda}(s)\right)_{\boldsymbol{H}_{0}} \\
& =\int_{\Omega} \beta_{\lambda}\left(u_{\varepsilon, \lambda}(s)\right)\left(u_{\varepsilon, \lambda}(s)-m_{0}\right) d x+\int_{\Gamma} \beta_{\lambda}\left(u_{\Gamma, \varepsilon, \lambda}(s)\right)\left(u_{\Gamma, \varepsilon, \lambda}(s)-m_{0}\right) d \Gamma \\
& \geq \int_{\Omega} \widehat{\beta}_{\lambda}\left(u_{\varepsilon, \lambda}(s)\right) d x-\widehat{\beta}_{\lambda}\left(m_{0}\right)|\Omega|+\int_{\Gamma} \widehat{\beta}_{\lambda}\left(u_{\Gamma, \varepsilon, \lambda}(s)\right) d \Gamma-\widehat{\beta}_{\lambda}\left(m_{0}\right)|\Gamma|
\end{aligned}
$$

for a.a. $s \in(0, T)$. Next, recalling the fundamental property of the chain rule with (2.6) and using the Young inequality, we have

$$
\begin{aligned}
& -\varepsilon\left\langle\boldsymbol{v}_{\varepsilon, \lambda}(s), \boldsymbol{P} \boldsymbol{\pi}\left(\boldsymbol{v}_{\varepsilon, \lambda}(s)+m_{0} \mathbf{1}\right)\right\rangle_{\boldsymbol{V}_{0}^{*}, \boldsymbol{V}_{0}} \\
& \leq \frac{1}{4}\left|\boldsymbol{v}_{\varepsilon, \lambda}(s)\right|_{\boldsymbol{V}_{0}^{*}}^{2}+\varepsilon^{2} \int_{\Omega}\left|\nabla \pi\left(v_{\varepsilon, \lambda}(s)+m_{0}\right)\right|^{2} d x+\varepsilon^{2} \int_{\Gamma}\left|\nabla_{\Gamma} \pi\left(v_{\Gamma, \varepsilon, \lambda}(s)+m_{0}\right)\right|^{2} d \Gamma \\
& \leq \frac{1}{4}\left|\boldsymbol{v}_{\varepsilon, \lambda}(s)\right|_{\boldsymbol{V}_{0}^{*}}^{2}+\varepsilon^{2}\left|\boldsymbol{v}_{\varepsilon, \lambda}(s)\right|_{\boldsymbol{V}_{0}}^{2},
\end{aligned}
$$

and

$$
\left\langle\boldsymbol{v}_{\varepsilon, \lambda}(s), \boldsymbol{f}(s)\right\rangle_{\boldsymbol{V}_{0}^{*}, \boldsymbol{V}_{0}} \leq \frac{1}{4}\left|\boldsymbol{v}_{\varepsilon, \lambda}(s)\right|_{\boldsymbol{V}_{0}^{*}}^{2}+|\boldsymbol{f}(s)|_{\boldsymbol{V}_{0}}^{2}
$$


for a.a. $s \in(0, T)$. Thus, correcting (3.1) (3.4), recalling the definition of the subdifferential and using (2.21), (2.24), and $\varphi(\mathbf{0})=0$, we see that

$$
\begin{aligned}
& \lambda \frac{d}{d s}\left|\boldsymbol{v}_{\varepsilon, \lambda}(s)\right|_{\boldsymbol{H}_{0}}^{2}+\frac{d}{d s}\left|\boldsymbol{v}_{\varepsilon, \lambda}(s)\right|_{V_{0}^{*}}^{2}+\left(\varepsilon-2 \varepsilon^{2}\right)\left|\boldsymbol{v}_{\varepsilon, \lambda}(s)\right|_{\boldsymbol{V}_{0}}^{2} \\
& \quad+2\left|\widehat{\beta}_{\lambda}\left(u_{\varepsilon, \lambda}(s)\right)\right|_{L^{1}(\Omega)}+2\left|\widehat{\beta}_{\lambda}\left(u_{\Gamma, \varepsilon, \lambda}(s)\right)\right|_{L^{1}(\Gamma)} \\
& \leq 2(|\Omega|+|\Gamma|) \widehat{\beta}\left(m_{0}\right)+\left|\boldsymbol{v}_{\varepsilon, \lambda}(s)\right|_{\boldsymbol{V}_{0}^{*}}^{2}+2|\boldsymbol{f}(s)|_{\boldsymbol{V}_{0}}^{2}
\end{aligned}
$$

for a.a. $s \in(0, T)$. If we take $\varepsilon \in(0,1 / 4]$, then $\varepsilon-2 \varepsilon^{2} \geq \varepsilon / 2>0$. By virtue of the Gronwall inequality, we obtain

$$
\begin{aligned}
& \lambda\left|\boldsymbol{v}_{\varepsilon, \lambda}(t)\right|_{\boldsymbol{H}_{0}}^{2}+\left|\boldsymbol{v}_{\varepsilon, \lambda}(t)\right|_{\boldsymbol{V}_{0}^{*}}^{2} \\
& \leq\left(\left|\boldsymbol{v}_{0}\right|_{\boldsymbol{H}_{0}}^{2}+\left|\boldsymbol{v}_{0}\right|_{\boldsymbol{V}_{0}^{*}}^{2}+2 T(|\Omega|+|\Gamma|) \widehat{\beta}\left(m_{0}\right)+2|\boldsymbol{f}|_{L^{2}\left(0, T ; \boldsymbol{V}_{0}\right)}^{2}\right) e^{T}=: M_{1}
\end{aligned}
$$

for all $t \in[0, T]$. Next, integrating (3.5) over $(0, t)$ with respect to $s$, we obtain

$$
\begin{aligned}
& \frac{\varepsilon}{2} \int_{0}^{t}\left|\boldsymbol{v}_{\varepsilon, \lambda}(s)\right|_{\boldsymbol{V}_{0}}^{2} d s+2 \int_{0}^{t}\left|\widehat{\beta}_{\lambda}\left(u_{\varepsilon, \lambda}(s)\right)\right|_{L^{1}(\Omega)} d s+2 \int_{0}^{t}\left|\widehat{\beta}_{\lambda}\left(u_{\Gamma, \varepsilon, \lambda}(s)\right)\right|_{L^{1}(\Gamma)} d s \\
& \leq\left|\boldsymbol{v}_{0}\right|_{\boldsymbol{H}_{0}}^{2}+\left|\boldsymbol{v}_{0}\right|_{\boldsymbol{V}_{0}^{*}}^{2}+2 T(|\Omega|+|\Gamma|) \widehat{\beta}\left(m_{0}\right)+2|\boldsymbol{f}|_{L^{2}\left(0, T ; \boldsymbol{V}_{0}\right)}^{2}+\int_{0}^{t}\left|\boldsymbol{v}_{\varepsilon, \lambda}(s)\right|_{\boldsymbol{V}_{0}^{*}}^{2} d s \\
& \leq M_{1}(1+T):=M_{2}
\end{aligned}
$$

for all $t \in[0, T]$. Thus, we have the conclusion.

Lemma 3.2. There exists a positive constant $M_{3}$, independent of $\varepsilon \in(0,1 / 4]$ and $\lambda \in$ $(0,1]$, such that

$$
\begin{gathered}
2 \lambda \int_{0}^{t}\left|\boldsymbol{v}_{\varepsilon, \lambda}^{\prime}(s)\right|_{\boldsymbol{H}_{0}}^{2} d s+\int_{0}^{t}\left|\boldsymbol{v}_{\varepsilon, \lambda}^{\prime}(s)\right|_{\boldsymbol{V}_{0}^{*}}^{2} d s+\varepsilon\left|\boldsymbol{v}_{\varepsilon, \lambda}(t)\right|_{\boldsymbol{V}_{0}}^{2} \\
+2\left|\widehat{\beta}_{\lambda}\left(u_{\varepsilon, \lambda}(t)\right)\right|_{L^{1}(\Omega)}+2\left|\widehat{\beta}_{\lambda}\left(u_{\Gamma, \varepsilon, \lambda}(t)\right)\right|_{L^{1}(\Gamma)} \leq M_{3}, \\
\int_{0}^{t}\left|\boldsymbol{P} \boldsymbol{\mu}_{\varepsilon, \lambda}(s)\right|_{\boldsymbol{V}_{0}}^{2} d s \leq M_{3}
\end{gathered}
$$

for all $t \in[0, T]$.

Proof. We test (2.22) at time $s \in(0, T)$ by $\boldsymbol{v}_{\varepsilon, \lambda}^{\prime}(s) \in \boldsymbol{H}_{0}$. Then, using the same method as for (3.3), we have

$$
-\varepsilon\left\langle\boldsymbol{v}_{\varepsilon, \lambda}^{\prime}(s), \boldsymbol{P} \boldsymbol{\pi}\left(\boldsymbol{v}_{\varepsilon, \lambda}(s)+m_{0} \mathbf{1}\right)\right\rangle_{\boldsymbol{V}_{0}^{*}, \boldsymbol{V}_{0}} \leq \frac{1}{4}\left|\boldsymbol{v}_{\varepsilon, \lambda}^{\prime}(s)\right|_{\boldsymbol{V}_{0}^{*}}^{2}+\varepsilon^{2}\left|\boldsymbol{v}_{\varepsilon, \lambda}(s)\right|_{\boldsymbol{V}_{0}}^{2},
$$

and

$$
\left\langle\boldsymbol{v}_{\varepsilon, \lambda}^{\prime}(s), \boldsymbol{f}(s)\right\rangle_{\boldsymbol{V}_{0}^{*}, \boldsymbol{V}_{0}} \leq \frac{1}{4}\left|\boldsymbol{v}_{\varepsilon, \lambda}^{\prime}(s)\right|_{\boldsymbol{V}_{0}^{*}}^{2}+|\boldsymbol{f}(s)|_{\boldsymbol{V}_{0}}^{2}
$$


for a.a. $s \in(0, T)$. Then, using (2.3) and the chain rule, we deduce

$$
\begin{aligned}
& \lambda\left|\boldsymbol{v}_{\varepsilon, \lambda}^{\prime}(s)\right|_{\boldsymbol{H}_{0}}^{2}+\frac{1}{2}\left|\boldsymbol{v}_{\varepsilon, \lambda}^{\prime}(s)\right|_{\boldsymbol{V}_{0}^{*}}^{2}+\varepsilon \frac{d}{d s} \varphi\left(\boldsymbol{v}_{\varepsilon, \lambda}(s)\right) \\
& \quad+\frac{d}{d s} \int_{\Omega} \widehat{\beta}_{\lambda}\left(v_{\varepsilon, \lambda}(s)+m_{0}\right) d x+\frac{d}{d s} \int_{\Gamma} \widehat{\beta}_{\lambda}\left(v_{\Gamma, \varepsilon, \lambda}(s)+m_{0}\right) d \Gamma \\
& \leq \varepsilon^{2}\left|\boldsymbol{v}_{\varepsilon, \lambda}(s)\right|_{\boldsymbol{V}_{0}}^{2}+|\boldsymbol{f}(s)|_{\boldsymbol{V}_{0}}^{2}
\end{aligned}
$$

for a.a. $s \in(0, T)$. Integrating (3.8) over $(0, t)$ with respect to $s$, we can find a positive constant $M_{3}$, depending only on $\left|\boldsymbol{v}_{0}\right|_{\boldsymbol{V}_{0}},\left|\widehat{\beta}\left(u_{0}\right)\right|_{L^{1}(\Omega)},\left|\widehat{\beta}\left(u_{0 \Gamma}\right)\right|_{L^{1}(\Gamma)}, M_{2}$, and $|\boldsymbol{f}|_{L^{2}\left(0, T ; \boldsymbol{V}_{0}\right)}$, such that the aforementioned estimate (3.6) holds. Next, to obtain (3.7), we recall (2.19), (see also Remark 3). We have

$$
\boldsymbol{\mu}_{\varepsilon, \lambda}(s)=\lambda \boldsymbol{v}_{\varepsilon, \lambda}^{\prime}(s)+\varepsilon \partial \varphi\left(\boldsymbol{v}_{\varepsilon, \lambda}(s)\right)+\boldsymbol{\beta}_{\lambda}\left(\boldsymbol{u}_{\varepsilon, \lambda}(s)\right)+\varepsilon \boldsymbol{\pi}\left(\boldsymbol{u}_{\varepsilon, \lambda}(s)\right)-\boldsymbol{f}(s) \quad \text { in } \boldsymbol{V},
$$

for a.a. $s \in(0, T)$. Therefore, the evolution equation (2.22) is equivalent to

$$
\boldsymbol{v}_{\varepsilon, \lambda}^{\prime}(s)+\boldsymbol{F}\left(\boldsymbol{P} \boldsymbol{\mu}_{\varepsilon, \lambda}(s)\right)=\mathbf{0} \quad \text { in } \boldsymbol{V}_{0}^{*}, \quad \text { for a.a. } s \in(0, T),
$$

with (3.9). We test (3.10) by $\boldsymbol{P} \boldsymbol{\mu}_{\varepsilon, \lambda}(s) \in \boldsymbol{V}_{0}$, and integrate the resultant over $(0, t)$ with respect to $s$. Then, using (2.2) and the Young inequality, we have

$$
\int_{0}^{t}\left|\boldsymbol{P} \boldsymbol{\mu}_{\varepsilon, \lambda}(s)\right|_{\boldsymbol{V}_{0}}^{2} d s \leq \frac{1}{2} \int_{0}^{t}\left|\boldsymbol{v}_{\varepsilon, \lambda}^{\prime}(s)\right|_{\boldsymbol{V}_{0}^{*}}^{2} d s+\frac{1}{2} \int_{0}^{t}\left|\boldsymbol{P} \boldsymbol{\mu}_{\varepsilon, \lambda}(s)\right|_{\boldsymbol{V}_{0}}^{2} d s
$$

for all $t \in[0, T]$. Thus, using (3.6), we obtain (3.7).

Lemma 3.3. There exist two positive constants $M_{4}$ and $M_{5}$, independent of $\varepsilon \in(0,1 / 4]$ and $\lambda \in(0,1]$, such that

$$
\begin{gathered}
\left|\boldsymbol{u}_{\varepsilon, \lambda}(t)\right|_{\boldsymbol{H}}^{2} \leq M_{4}, \quad\left|\boldsymbol{v}_{\varepsilon, \lambda}(t)\right|_{\boldsymbol{H}_{0}}^{2} \leq M_{4} \\
\int_{0}^{t}\left|\beta_{\lambda}\left(u_{\varepsilon, \lambda}(s)\right)\right|_{L^{1}(\Omega)}^{2} d s+\int_{0}^{t}\left|\beta_{\lambda}\left(u_{\Gamma, \varepsilon, \lambda}(s)\right)\right|_{L^{1}(\Gamma)}^{2} d s \leq M_{5}
\end{gathered}
$$

for all $t \in[0, T]$.

Proof. From (2.5) in (A1) and (2.20),

$$
\begin{aligned}
\widehat{\beta}_{\lambda}(r) & =\frac{1}{2 \lambda}\left|r-J_{\lambda}(r)\right|^{2}+\widehat{\beta}\left(J_{\lambda}(r)\right) \\
& \geq \frac{1}{2 \lambda}\left|r-J_{\lambda}(r)\right|^{2}+c_{1}\left|J_{\lambda}(r)\right|^{2}-c_{2} \quad \text { for all } r \in \mathbb{R} .
\end{aligned}
$$

Therefore, from (3.6) in Lemma 3.2, we obtain

$$
\begin{gathered}
\frac{1}{2 \lambda}\left|u_{\varepsilon, \lambda}(t)-J_{\lambda}\left(u_{\varepsilon, \lambda}(t)\right)\right|_{H}^{2}+c_{1}\left|J_{\lambda}\left(u_{\varepsilon, \lambda}(t)\right)\right|_{H}^{2} \leq \frac{M_{3}}{2}+c_{2}|\Omega|, \\
\frac{1}{2 \lambda}\left|u_{\Gamma, \varepsilon, \lambda}(t)-J_{\lambda}\left(u_{\Gamma, \varepsilon, \lambda}(t)\right)\right|_{H_{\Gamma}}^{2}+c_{1}\left|J_{\lambda}\left(u_{\Gamma, \varepsilon, \lambda}(t)\right)\right|_{H_{\Gamma}}^{2} \leq \frac{M_{3}}{2}+c_{2}|\Gamma|
\end{gathered}
$$


for all $t \in[0, T]$. Thanks to this $\lambda \in(0,1]$ implies that there exists $\tilde{M}_{4}>0$, depending only on $c_{1}, c_{2}, M_{3},|\Omega|$, and $|\Gamma|$, such that

$$
\begin{aligned}
\left|\boldsymbol{u}_{\varepsilon, \lambda}(t)\right|_{\boldsymbol{H}}^{2}= & \left|u_{\varepsilon, \lambda}(t)\right|_{H}^{2}+\left|u_{\Gamma, \varepsilon, \lambda}(t)\right|_{H_{\Gamma}}^{2} \\
\leq & 2\left|u_{\varepsilon, \lambda}(t)-J_{\lambda}\left(u_{\varepsilon, \lambda}(t)\right)\right|_{H}^{2}+2\left|J_{\lambda}\left(u_{\varepsilon, \lambda}(t)\right)\right|_{H}^{2} \\
& +2\left|u_{\Gamma, \varepsilon, \lambda}(t)-J_{\lambda}\left(u_{\Gamma, \varepsilon, \lambda}(t)\right)\right|_{H_{\Gamma}}^{2}+2\left|J_{\lambda}\left(u_{\Gamma, \varepsilon, \lambda}(t)\right)\right|_{H_{\Gamma}}^{2} \\
\leq & \tilde{M}_{4}
\end{aligned}
$$

for all $t \in[0, T]$. Then, there exists $M_{4}>0$, depending only on $\tilde{M}_{4},\left|m_{0}\right|,|\Omega|$, and $|\Gamma|$, such that

$$
\begin{aligned}
\left|\boldsymbol{v}_{\varepsilon, \lambda}(t)\right|_{\boldsymbol{H}}^{2} & \leq 2\left|\boldsymbol{u}_{\varepsilon, \lambda}(t)\right|_{\boldsymbol{H}}^{2}+2\left|m_{0}\right|^{2}(|\Omega|+|\Gamma|) \\
& \leq M_{4}
\end{aligned}
$$

for all $t \in[0, T]$. Thus, (3.11) has been obtained. Next, recall the useful inequality [18, Section 5]. Indeed, from assumption (A4), we have $m_{0} \in \operatorname{int} D(\beta)$ (which is the criterion needed to apply the inequality), meaning there exist two constants $c_{3}, c_{4}>0$ such that

$$
\beta_{\lambda}(r)\left(r-m_{0}\right) \geq c_{3} \beta_{\lambda}(r)-c_{4}
$$

for all $r \in \mathbb{R}$ and $\lambda \in(0,1]$. Then, (3.2) can be improved by

$$
\begin{aligned}
& \left(\boldsymbol{P} \boldsymbol{\beta}_{\lambda}\left(\boldsymbol{v}_{\varepsilon, \lambda}(s)+m_{0} \mathbf{1}\right), \boldsymbol{v}_{\varepsilon, \lambda}(s)\right)_{\boldsymbol{H}_{0}} \\
& =\int_{\Omega} \beta_{\lambda}\left(u_{\varepsilon, \lambda}(s)\right)\left(u_{\varepsilon, \lambda}(s)-m_{0}\right) d x+\int_{\Gamma} \beta_{\lambda}\left(u_{\Gamma, \varepsilon, \lambda}(s)\right)\left(u_{\Gamma, \varepsilon, \lambda}(s)-m_{0}\right) d \Gamma \\
& \geq c_{3} \int_{\Omega} \beta_{\lambda}\left(u_{\varepsilon, \lambda}(s)\right) d x-c_{4}|\Omega|+c_{3} \int_{\Gamma} \beta_{\lambda}\left(u_{\Gamma, \varepsilon, \lambda}(s)\right) d \Gamma-c_{4}|\Gamma|
\end{aligned}
$$

for a.a. $s \in(0, T)$. Therefore, by using (2.3) and the monotonicity of $\partial \varphi$,

$$
\begin{aligned}
& c_{3} \int_{\Omega} \beta_{\lambda}\left(u_{\varepsilon, \lambda}(s)\right) d x+c_{3} \int_{\Gamma} \beta_{\lambda}\left(u_{\Gamma, \varepsilon, \lambda}(s)\right) d \Gamma \\
& \leq c_{4}(|\Omega|+|\Gamma|)+\lambda\left|\boldsymbol{v}_{\varepsilon, \lambda}^{\prime}(s)\right|_{\boldsymbol{H}_{0}}\left|\boldsymbol{v}_{\varepsilon, \lambda}(s)\right|_{\boldsymbol{H}_{0}}+\left|\boldsymbol{v}_{\varepsilon, \lambda}^{\prime}(s)\right|_{\boldsymbol{V}_{0}^{*}}\left|\boldsymbol{v}_{\varepsilon, \lambda}(s)\right|_{\boldsymbol{V}_{0}^{*}} \\
& \quad+\varepsilon\left|\boldsymbol{\pi}\left(\boldsymbol{u}_{\varepsilon, \lambda}(s)\right)\right|_{\boldsymbol{H}}\left|\boldsymbol{v}_{\varepsilon, \lambda}(s)\right|_{\boldsymbol{H}_{0}}+|\boldsymbol{f}(s)|_{\boldsymbol{H}_{0}}\left|\boldsymbol{v}_{\varepsilon, \lambda}(s)\right|_{\boldsymbol{H}_{0}}
\end{aligned}
$$

for a.a. $s \in(0, T)$. Now, squaring the above and using Lemma 3.1 and (3.11), we obtain

$$
\begin{aligned}
& \left(c_{3} \int_{\Omega} \beta_{\lambda}\left(u_{\varepsilon, \lambda}(s)\right) d x+c_{3} \int_{\Gamma} \beta_{\lambda}\left(u_{\Gamma, \varepsilon, \lambda}(s)\right) d \Gamma\right)^{2} \\
& \leq 5 c_{4}^{2}(|\Omega|+|\Gamma|)^{2}+5 \lambda^{2} M_{4}\left|\boldsymbol{v}_{\varepsilon, \lambda}^{\prime}(s)\right|_{\boldsymbol{H}_{0}}^{2}+5 M_{1}\left|\boldsymbol{v}_{\varepsilon, \lambda}^{\prime}(s)\right|_{\boldsymbol{V}_{0}^{*}}^{2} \\
& \quad+5 \varepsilon^{2} M_{4}\left|\boldsymbol{\pi}\left(\boldsymbol{u}_{\varepsilon, \lambda}(s)\right)\right|_{\boldsymbol{H}}^{2}+5 M_{4}|\boldsymbol{f}(s)|_{\boldsymbol{H}_{0}}^{2}
\end{aligned}
$$


for a.a. $s \in(0, T)$. Thus, from (2.6) and (3.11), we have

$$
\begin{aligned}
\left|\boldsymbol{\pi}\left(\boldsymbol{u}_{\varepsilon, \lambda}(s)\right)\right|_{\boldsymbol{H}}^{2} & =\left|\boldsymbol{\pi}\left(\boldsymbol{u}_{\varepsilon, \lambda}(s)\right)-\boldsymbol{\pi}\left(m_{0} \mathbf{1}\right)+\boldsymbol{\pi}\left(m_{0} \mathbf{1}\right)\right|_{\boldsymbol{H}}^{2} \\
& \leq 2\left\{\int_{\Omega}\left(\left|v_{\varepsilon, \lambda}(s)\right|^{2}+\left|\pi\left(m_{0}\right)\right|^{2}\right) d x+\int_{\Gamma}\left(\left|v_{\Gamma, \varepsilon, \lambda}(s)\right|^{2}+\left|\pi\left(m_{0}\right)\right|^{2}\right) d \Gamma\right\} \\
& \leq 2\left\{\left|\boldsymbol{v}_{\varepsilon, \lambda}(s)\right|_{\boldsymbol{H}_{0}}^{2}+\left|\pi\left(m_{0}\right)\right|^{2}(|\Omega|+|\Gamma|)\right\} \\
& \leq 2\left\{M_{4}+\left|\pi\left(m_{0}\right)\right|^{2}(|\Omega|+|\Gamma|)\right\}=: \tilde{M}_{5}
\end{aligned}
$$

for a.a. $s \in(0, T)$. We integrate the resultant (3.14) over $(0, t)$ with respect to $s$. From Lemma 3.2, there exists a positive constant $M_{5}$, depending only on $c_{3}, c_{4}, T,|\Omega|,|\Gamma|, M_{1}$, $M_{3}, M_{4}, \tilde{M}_{5}$, and $|\boldsymbol{f}|_{L^{2}\left(0, T ; \boldsymbol{H}_{0}\right)}$ and independent of $\varepsilon \in(0,1 / 4]$ and $\lambda \in(0,1]$, such that (3.12) holds.

Now, recalling (3.9) and using the fact that $\boldsymbol{v}_{\varepsilon, \lambda}^{\prime}(t), \partial \varphi\left(\boldsymbol{v}_{\varepsilon, \lambda}(t)\right) \in \boldsymbol{H}_{0}$ for a.a. $t \in(0, T)$, we obtain

$$
m\left(\boldsymbol{\mu}_{\varepsilon, \lambda}(t)\right)=m\left(\boldsymbol{\beta}_{\lambda}\left(\boldsymbol{u}_{\varepsilon, \lambda}(t)\right)+\varepsilon \boldsymbol{\pi}\left(\boldsymbol{u}_{\varepsilon, \lambda}(t)\right)-\boldsymbol{f}(t)\right) \quad \text { for a.a. } t \in(0, T) .
$$

Lemma 3.4. There exist two positive constants $M_{6}$ and $M_{7}$, independent of $\varepsilon \in(0,1 / 4]$ and $\lambda \in(0,1]$, such that

$$
\begin{gathered}
\int_{0}^{t}\left|m\left(\boldsymbol{\mu}_{\varepsilon, \lambda}(s)\right)\right|^{2} d s \leq M_{6} \\
\int_{0}^{t}\left|\boldsymbol{\mu}_{\varepsilon, \lambda}(s)\right|_{\boldsymbol{V}}^{2} d s \leq M_{7}
\end{gathered}
$$

for all $t \in[0, T]$.

Proof. From (2.1) and (3.16), we have

$$
\begin{aligned}
\left|m\left(\boldsymbol{\mu}_{\varepsilon, \lambda}(s)\right)\right|^{2} & \leq \frac{6}{(|\Omega|+|\Gamma|)^{2}}\left\{\left|\beta_{\lambda}\left(u_{\varepsilon, \lambda}(s)\right)\right|_{L^{1}(\Omega)}^{2}+\varepsilon^{2}\left|\pi\left(u_{\varepsilon, \lambda}(s)\right)\right|_{L^{1}(\Omega)}^{2}+|\Omega||f(s)|_{H}^{2}\right. \\
& \left.+\left|\beta_{\lambda}\left(u_{\Gamma, \varepsilon, \lambda}(s)\right)\right|_{L^{1}(\Gamma)}^{2}+\left|\pi\left(u_{\Gamma, \varepsilon, \lambda}(s)\right)\right|_{L^{1}(\Gamma)}^{2}+|\Gamma|\left|f_{\Gamma}(s)\right|_{H_{\Gamma}}^{2}\right\}
\end{aligned}
$$

for a.a. $s \in(0, T)$. Then, by integrating over $(0, T)$, it follows that there is a positive constant $M_{6}$, depending only on $T,|\Omega|,|\Gamma|, M_{4}, M_{5},\left|\pi\left(m_{0}\right)\right|$, and $|\boldsymbol{f}|_{L^{2}\left(0, T ; \boldsymbol{H}_{0}\right)}$, such that the first estimate holds. Next, from the Poincare inequality (see, e.g., [11, Lemma A]), we know that there exists a positive constant $c_{p}$ such that

$$
c_{p}|\boldsymbol{z}|_{\boldsymbol{V}}^{2} \leq|\boldsymbol{z}|_{\boldsymbol{V}_{0}}^{2} \text { for all } \boldsymbol{z} \in \boldsymbol{V}_{0}
$$


TAKESHI FUKAO

Therefore,

$$
\begin{aligned}
\int_{0}^{t}\left|\boldsymbol{\mu}_{\varepsilon, \lambda}(s)\right|_{\boldsymbol{V}}^{2} d s & \leq 2 \int_{0}^{t}\left|\boldsymbol{P} \boldsymbol{\mu}_{\varepsilon, \lambda}(s)\right|_{\boldsymbol{V}}^{2} d s+2 \int_{0}^{t}\left|m\left(\boldsymbol{\mu}_{\varepsilon, \lambda}(s)\right) \mathbf{1}\right|_{\boldsymbol{V}}^{2} d s \\
& \leq \frac{2}{c_{p}} \int_{0}^{t}\left|\boldsymbol{P} \boldsymbol{\mu}_{\varepsilon, \lambda}(s)\right|_{\boldsymbol{V}_{0}}^{2} d s+2(|\Omega|+|\Gamma|) \int_{0}^{t}\left|m\left(\boldsymbol{\mu}_{\varepsilon, \lambda}(s)\right)\right|^{2} d s \\
& \leq \frac{2 M_{3}}{c_{p}}+2(|\Omega|+|\Gamma|) M_{6}=: M_{7}
\end{aligned}
$$

for all $t \in[0, T]$.

Lemma 3.5. There exists a positive constant $M_{8}$, independent of $\varepsilon \in(0,1 / 4]$ and $\lambda \in$ $(0,1]$, such that

$$
\int_{0}^{t}\left|\boldsymbol{\beta}_{\lambda}\left(\boldsymbol{u}_{\varepsilon, \lambda}(s)\right)\right|_{\boldsymbol{H}}^{2} d s \leq M_{8}
$$

for all $t \in[0, T]$.

Proof. We test (3.9) by $\boldsymbol{\beta}_{\lambda}\left(\boldsymbol{u}_{\varepsilon, \lambda}(s)\right) \in \boldsymbol{V}$, then

$$
\begin{aligned}
& \varepsilon\left(\partial \varphi\left(\boldsymbol{v}_{\varepsilon, \lambda}(s)\right), \boldsymbol{\beta}_{\lambda}\left(\boldsymbol{u}_{\varepsilon, \lambda}(s)\right)\right)_{\boldsymbol{H}}+\left|\boldsymbol{\beta}_{\lambda}\left(\boldsymbol{u}_{\varepsilon, \lambda}(s)\right)\right|_{\boldsymbol{H}}^{2} \\
& \leq\left(\boldsymbol{\mu}_{\varepsilon, \lambda}(s)-\lambda \boldsymbol{v}_{\varepsilon, \lambda}^{\prime}(s)-\varepsilon \boldsymbol{\pi}\left(\boldsymbol{u}_{\varepsilon, \lambda}(s)\right)+\boldsymbol{f}(s), \boldsymbol{\beta}_{\lambda}\left(\boldsymbol{u}_{\varepsilon, \lambda}(s)\right)\right)_{\boldsymbol{H}}
\end{aligned}
$$

for a.a. $s \in(0, T)$. Here, from the monotonicity of $\beta_{\lambda}$, we have

$$
\begin{array}{rl}
\varepsilon & \left(\partial \varphi\left(\boldsymbol{v}_{\varepsilon, \lambda}(s)\right), \boldsymbol{\beta}_{\lambda}\left(\boldsymbol{u}_{\varepsilon, \lambda}(s)\right)\right)_{\boldsymbol{H}} \\
= & \varepsilon \int_{\Omega}\left(-\Delta v_{\varepsilon, \lambda}(s)\right) \beta_{\lambda}\left(u_{\varepsilon, \lambda}(s)\right) d x+\varepsilon \int_{\Gamma} \partial_{\boldsymbol{\nu}} v_{\varepsilon, \lambda}(s) \beta_{\lambda}\left(u_{\Gamma, \varepsilon, \lambda}(s)\right) d \Gamma \\
& +\varepsilon \int_{\Gamma}\left(-\Delta_{\Gamma} v_{\Gamma, \varepsilon, \lambda}(s)\right) \beta_{\lambda}\left(u_{\Gamma, \varepsilon, \lambda}(s)\right) d \Gamma \\
= & \varepsilon \int_{\Omega} \beta_{\lambda}^{\prime}\left(u_{\varepsilon, \lambda}(s)\right)\left|\nabla v_{\varepsilon, \lambda}(s)\right|^{2} d x+\int_{\Gamma} \beta_{\lambda}^{\prime}\left(u_{\Gamma, \varepsilon, \lambda}(s)\right)\left|\nabla_{\Gamma} v_{\Gamma, \varepsilon, \lambda}(s)\right|^{2} d \Gamma \\
\geq 0 & 0
\end{array}
$$

for a.a. $s \in(0, T)$, where we have used the fact that $\beta_{\lambda}\left(u_{\varepsilon, \lambda}(s)\right)_{\left.\right|_{\Gamma}}=\beta_{\lambda}\left(u_{\Gamma, \varepsilon, \lambda}(s)\right)$ a.e. on $\Gamma$. Moreover, by the Young inequality,

$$
\begin{aligned}
& \left(\boldsymbol{\mu}_{\varepsilon, \lambda}(s)-\lambda \boldsymbol{v}_{\varepsilon, \lambda}^{\prime}(s)-\varepsilon \boldsymbol{\pi}\left(\boldsymbol{u}_{\varepsilon, \lambda}(s)\right)+\boldsymbol{f}(s), \boldsymbol{\beta}_{\lambda}\left(\boldsymbol{u}_{\varepsilon, \lambda}(s)\right)\right)_{\boldsymbol{H}} \\
& \leq \frac{1}{2}\left|\boldsymbol{\beta}_{\lambda}\left(\boldsymbol{u}_{\varepsilon, \lambda}(s)\right)\right|_{\boldsymbol{H}}^{2}+2\left|\boldsymbol{\mu}_{\varepsilon, \lambda}(s)\right|_{\boldsymbol{H}}^{2}+2 \lambda^{2}\left|\boldsymbol{v}_{\varepsilon, \lambda}^{\prime}(s)\right|_{\boldsymbol{H}_{0}}^{2}+2 \varepsilon^{2}\left|\boldsymbol{\pi}\left(\boldsymbol{u}_{\varepsilon, \lambda}(s)\right)\right|_{\boldsymbol{H}}^{2}+2|\boldsymbol{f}(s)|_{\boldsymbol{H}_{0}}^{2}
\end{aligned}
$$

for a.a. $s \in(0, T)$. Let us combineing the above inequalities, and integrateing in over $(0, T)$ with respect to $s$. Then, recalling (2.21) and (3.15), and using Lemmas 3.2 and 
3.4, we obtain

$$
\begin{aligned}
\int_{0}^{t}\left|\boldsymbol{\beta}_{\lambda}\left(\boldsymbol{u}_{\varepsilon, \lambda}(s)\right)\right|_{\boldsymbol{H}}^{2} d s \leq & 4 \int_{0}^{T}\left|\boldsymbol{\mu}_{\varepsilon, \lambda}(s)\right|_{\boldsymbol{H}}^{2} d s+4 \lambda^{2} \int_{0}^{T}\left|\boldsymbol{v}_{\varepsilon, \lambda}^{\prime}(s)\right|_{\boldsymbol{H}_{0}}^{2} d s \\
& +4 \varepsilon^{2} \int_{0}^{T}\left|\boldsymbol{\pi}\left(\boldsymbol{u}_{\varepsilon, \lambda}(s)\right)\right|_{\boldsymbol{H}}^{2} d s+4 \int_{0}^{T}|\boldsymbol{f}(s)|_{\boldsymbol{H}_{0}}^{2} d s \\
\leq & 4 M_{7}+4 M_{3}+4 T \tilde{M}_{5}+4|\boldsymbol{f}|_{L^{2}\left(0, T ; \boldsymbol{H}_{0}\right)}^{2}=: M_{8}
\end{aligned}
$$

for all $t \in[0, T]$.

\subsection{Uniform estimates for approximate solutions of $(\mathrm{P})_{\varepsilon}$}

In this subsection, based on the result of Proposition 2.1, we obtain uniform estimates for the solutions of $(\mathrm{P})_{\varepsilon}$. Actually, by obtaining additional uniform estimates (see [1] ) and passing to the limit in the approximate problem $(\mathrm{P})_{\varepsilon, \lambda}$ as $\lambda \rightarrow 0$, Proposition 2.1 can be proved. Thus, for each $\varepsilon \in(0,1 / 4]$, there exists a triplet $\left(\boldsymbol{v}_{\varepsilon}, \boldsymbol{\mu}_{\varepsilon}, \boldsymbol{\xi}_{\varepsilon}\right)$ of

$$
\begin{gathered}
\boldsymbol{v}_{\varepsilon} \in H^{1}\left(0, T ; \boldsymbol{V}_{0}^{*}\right) \cap L^{\infty}\left(0, T ; \boldsymbol{V}_{0}\right) \cap L^{2}(0, T ; \boldsymbol{W}), \\
\boldsymbol{\mu}_{\varepsilon} \in L^{2}(0, T ; \boldsymbol{V}), \quad \boldsymbol{\xi}_{\varepsilon} \in L^{2}(0, T ; \boldsymbol{H}), \quad \boldsymbol{\xi}_{\varepsilon} \in \boldsymbol{\beta}\left(\boldsymbol{u}_{\varepsilon}\right) \quad \text { in } L^{2}(0, T ; \boldsymbol{H})
\end{gathered}
$$

that satisfy (2.17), (2.18), and $\boldsymbol{v}_{\varepsilon}(0)=\boldsymbol{v}_{0}$ in $\boldsymbol{H}_{0}$. Here, we also have

$$
\boldsymbol{u}_{\varepsilon} \in H^{1}\left(0, T ; \boldsymbol{V}^{*}\right) \cap L^{\infty}(0, T ; \boldsymbol{V}) \cap L^{2}(0, T ; \boldsymbol{W}),
$$

with the relation $\boldsymbol{u}_{\varepsilon}=\boldsymbol{v}_{\varepsilon}+m_{0} \mathbf{1}$. On taking the limit $\lambda \rightarrow 0$, we obtain the same kind of uniform estimates as obtained in the previous lemmas, namely

$$
\begin{gathered}
\int_{0}^{t}\left|\boldsymbol{v}_{\varepsilon}^{\prime}(s)\right|_{\boldsymbol{V}_{0}^{*}}^{2} d s \leq M_{3}, \\
\varepsilon\left|\boldsymbol{v}_{\varepsilon}(t)\right|_{\boldsymbol{V}_{0}}^{2} \leq M_{3}, \\
\left|\boldsymbol{u}_{\varepsilon}(t)\right|_{\boldsymbol{H}}^{2} \leq M_{4}, \\
\left|\boldsymbol{v}_{\varepsilon}(t)\right|_{\boldsymbol{H}_{0}}^{2} \leq M_{4}, \\
\int_{0}^{t}\left|\boldsymbol{\mu}_{\varepsilon}(s)\right|_{\boldsymbol{V}}^{2} d s \leq M_{7}, \\
\int_{0}^{t}\left|\boldsymbol{\xi}_{\varepsilon}(s)\right|_{\boldsymbol{H}}^{2} d s \leq M_{8}
\end{gathered}
$$

for all $t \in[0, T]$. Using these estimates, we can prove the main theorem.

\section{Proof of the main theorem}

In this subsection, we conclude the proof of the main theorem by passing to the limit in the approximate problem $(\mathrm{P})_{\varepsilon}$ as $\varepsilon \rightarrow 0$. 
Proof of Theorem 2.1. By using the estimates (3.19)-(3.24) stated in the previous section, there exist a subsequence $\left\{\varepsilon_{k}\right\}_{k \in \mathbb{N}}$ with $\varepsilon_{k} \rightarrow 0$ as $k \rightarrow+\infty$ and some limit functions $\boldsymbol{u} \in H^{1}\left(0, T ; \boldsymbol{V}^{*}\right) \cap L^{\infty}(0, T ; \boldsymbol{H}), \boldsymbol{v} \in H^{1}\left(0, T ; \boldsymbol{V}_{0}^{*}\right) \cap L^{\infty}\left(0, T ; \boldsymbol{H}_{0}\right), \boldsymbol{\mu} \in L^{2}(0, T ; \boldsymbol{V})$, and $\boldsymbol{\xi} \in L^{2}(0, T ; \boldsymbol{H})$ such that

$$
\begin{gathered}
\boldsymbol{v}_{\varepsilon_{k}} \rightarrow \boldsymbol{v} \quad \text { weakly star in } L^{\infty}\left(0, T ; \boldsymbol{H}_{0}\right), \\
\boldsymbol{u}_{\varepsilon_{k}} \rightarrow \boldsymbol{u}=\boldsymbol{v}+m_{0} \mathbf{1} \quad \text { weakly star in } L^{\infty}(0, T ; \boldsymbol{H}), \\
\varepsilon_{k} \boldsymbol{v}_{\varepsilon_{k}} \rightarrow \mathbf{0} \quad \text { in } L^{\infty}\left(0, T ; \boldsymbol{V}_{0}\right), \\
\boldsymbol{v}_{\varepsilon_{k}}^{\prime} \rightarrow \boldsymbol{v}^{\prime} \quad \text { weakly in } L^{2}\left(0, T ; \boldsymbol{V}_{0}^{*}\right), \\
\boldsymbol{u}_{\varepsilon_{k}}^{\prime} \rightarrow \boldsymbol{u}^{\prime} \quad \text { weakly in } L^{2}\left(0, T ; \boldsymbol{V}^{*}\right), \\
\boldsymbol{\mu}_{\varepsilon_{k}} \rightarrow \boldsymbol{\mu} \quad \text { weakly in } L^{2}(0, T ; \boldsymbol{V}), \\
\boldsymbol{\xi}_{\varepsilon_{k}} \rightarrow \boldsymbol{\xi} \quad \text { weakly in } L^{2}(0, T ; \boldsymbol{H}), \\
\varepsilon_{k} \boldsymbol{\pi}\left(\boldsymbol{u}_{\varepsilon_{k}}\right) \rightarrow \mathbf{0} \text { in } L^{\infty}(0, T ; \boldsymbol{H})
\end{gathered}
$$

as $k \rightarrow+\infty$. From (4.25), (4.26), (4.28), and (4.29), the well-known Ascoli-Arzela theorem (see, e.g., [26, Section 8, Corollary 4]) gives

$$
\begin{aligned}
& \boldsymbol{v}_{\varepsilon_{k}} \rightarrow \boldsymbol{v} \text { in } C\left([0, T] ; \boldsymbol{V}_{0}^{*}\right), \\
& \boldsymbol{u}_{\varepsilon_{k}} \rightarrow \boldsymbol{u} \text { in } C\left([0, T] ; \boldsymbol{V}^{*}\right)
\end{aligned}
$$

as $k \rightarrow+\infty$. Now, integrating (2.18) over $(0, T)$, we find

$$
\begin{aligned}
\int_{0}^{T}\left(\boldsymbol{\mu}_{\varepsilon_{k}}(t), \boldsymbol{\eta}(t)\right)_{\boldsymbol{H}} d t & =\varepsilon_{k} \int_{0}^{T} a\left(\boldsymbol{v}_{\varepsilon_{k}}(t), \boldsymbol{\eta}(t)\right) d t+\int_{0}^{T}\left(\boldsymbol{\xi}_{\varepsilon_{k}}(t), \boldsymbol{\eta}(t)\right)_{\boldsymbol{H}} d t \\
& +\varepsilon_{k} \int_{0}^{T}\left(\boldsymbol{\pi}\left(\boldsymbol{u}_{\varepsilon_{k}}(t)\right), \boldsymbol{\eta}(t)\right)_{\boldsymbol{H}} d t-\int_{0}^{T}(\boldsymbol{f}(t), \boldsymbol{\eta}(t))_{\boldsymbol{H}} d t \\
& \text { for all } \boldsymbol{\eta} \in L^{2}(0, T ; \boldsymbol{V}) .
\end{aligned}
$$

Letting $k \rightarrow \infty$, we can use (4.30), (4.27), (4.31), and (4.32) to obtain

$$
\int_{0}^{T}(\boldsymbol{\mu}(t), \boldsymbol{\eta}(t))_{\boldsymbol{H}} d t=\int_{0}^{T}(\boldsymbol{\xi}(t)-\boldsymbol{f}(t), \boldsymbol{\eta}(t))_{\boldsymbol{H}} d t \quad \text { for all } \boldsymbol{\eta} \in L^{2}(0, T ; \boldsymbol{V}),
$$

namely, $\boldsymbol{\mu}=\boldsymbol{\xi}-\boldsymbol{f}$ in $L^{2}(0, T ; \boldsymbol{H})$. Additionally, the information $\boldsymbol{\mu}+\boldsymbol{f} \in L^{2}(0, T ; \boldsymbol{V})$ gives the regularity of $\boldsymbol{\xi} \in L^{2}(0, T ; \boldsymbol{V})$, namely $\xi_{\Gamma}=\xi_{\left.\right|_{\Gamma}}$ a.e. on $\Sigma$. Next, we take $\boldsymbol{\eta}:=$ $\boldsymbol{u}_{\varepsilon_{k}} \in L^{2}(0, T ; \boldsymbol{V})$ in (4.35). Then, using the positivity

$$
\varepsilon_{k} \int_{0}^{T} a\left(\boldsymbol{v}_{\varepsilon_{k}}(t), \boldsymbol{u}_{\varepsilon_{k}}(t)\right) d t=\varepsilon_{k} \int_{0}^{T}\left|\boldsymbol{v}_{\varepsilon_{k}}(t)\right|_{\boldsymbol{V}}^{2} d t \geq 0,
$$

and recalling (4.26), (4.30), (4.32), and (4.34), we have

$$
\begin{aligned}
\limsup _{k \rightarrow+\infty} \int_{0}^{T}\left(\boldsymbol{\xi}_{\varepsilon_{k}}(t), \boldsymbol{u}_{\varepsilon_{k}}(t)\right)_{\boldsymbol{H}} d t & \leq \int_{0}^{T}\langle\boldsymbol{u}(t), \boldsymbol{\mu}(t)\rangle_{\boldsymbol{V}^{*}, \boldsymbol{V}} d t+\int_{0}^{T}(\boldsymbol{f}(t), \boldsymbol{u}(t))_{\boldsymbol{H}} d t \\
& =\int_{0}^{T}(\boldsymbol{\xi}(t), \boldsymbol{u}(t))_{\boldsymbol{H}} d t .
\end{aligned}
$$


Thus, applying [4, Proposition 2.2, p. 38] with (4.25) and (4.31), we can deduce that

$$
\boldsymbol{\xi} \in \boldsymbol{\beta}(\boldsymbol{u}) \text { a.e. in } Q
$$

from the maximal monotonicity of $\beta$. Namely, we obtain $\xi \in \beta(u)$ a.e. in $\Omega, \xi_{\Gamma} \in \beta\left(u_{\Gamma}\right)$ a.e. on $\Gamma$. Finally, integrating (2.17) over $(0, T)$ with respect to $t$ and letting $k \rightarrow+\infty$, we have

$$
\int_{0}^{T}\left\langle\boldsymbol{v}^{\prime}(t), \boldsymbol{\eta}(t)\right\rangle_{\boldsymbol{V}_{0}^{*}, \boldsymbol{V}_{0}} d t+\int_{0}^{T} a(\boldsymbol{P} \boldsymbol{\mu}(t), \boldsymbol{\eta}(t)) d t=0 \quad \text { for all } \boldsymbol{\eta} \in L^{2}\left(0, T ; \boldsymbol{V}_{0}\right) .
$$

Next, using (2.2), we deduce

$$
\boldsymbol{v}^{\prime}(t)+\boldsymbol{F}(\boldsymbol{P} \boldsymbol{\mu}(t))=\mathbf{0} \quad \text { in } \boldsymbol{V}_{0}^{*}, \quad \text { for a.a. } t \in(0, T),
$$

namely

$$
\left\langle\boldsymbol{v}^{\prime}(t), \boldsymbol{z}\right\rangle_{\boldsymbol{V}_{0}^{*}, \boldsymbol{V}_{0}}+a(\boldsymbol{\xi}(t), \boldsymbol{z})=a(\boldsymbol{f}(t), \boldsymbol{z}) \quad \text { for all } \boldsymbol{z} \in \boldsymbol{V}_{0} .
$$

Therefore, recalling (2.16) and [11, Remark 2], we finally obtain

$$
\begin{aligned}
\left\langle u^{\prime}(t), z\right\rangle_{V^{*}, V} & +\left\langle u_{\Gamma}^{\prime}(t), z_{\Gamma}\right\rangle_{V_{\Gamma}^{*}, V_{\Gamma}}+\int_{\Omega} \nabla \xi(t) \cdot \nabla z d x+\int_{\Gamma} \nabla_{\Gamma} \xi_{\Gamma}(t) \cdot \nabla_{\Gamma} z_{\Gamma} d \Gamma \\
& =\int_{\Omega} g(t) z d x+\int_{\Gamma} g_{\Gamma}(t) z_{\Gamma} d \Gamma \quad \text { for all } \boldsymbol{z}=\left(z, z_{\Gamma}\right) \in \boldsymbol{V},
\end{aligned}
$$

for a.a. $t \in(0, T)$, with $u(0)=u_{0}$ a.e. in $\Omega$ and $u_{\Gamma}(0)=u_{0 \Gamma}$ a.e. on $\Gamma$. Thus, it turns out that the pair $(\boldsymbol{u}, \boldsymbol{\xi})$ is a weak solution of $(\mathrm{P})$.

\section{Continuous dependence}

In this section, we prove the continuous dependence of the data. This theorem also guarantees the uniqueness of the component $\boldsymbol{u}$ in the solution.

Proof of Theorem 2.2. For $i=1,2$, let $\left(\boldsymbol{u}^{(i)}, \boldsymbol{\xi}^{(i)}\right)$ be a weak solution of (P) corresponding to the data $\left(\boldsymbol{f}^{(i)}, \boldsymbol{u}_{0}^{(i)}\right)$. Set $m_{0}^{*}:=m\left(\boldsymbol{u}_{0}^{(1)}\right)=m\left(\boldsymbol{u}_{0}^{(2)}\right)$. From the weak formulation (4.36) of the Stefan problem (P) for $\boldsymbol{v}^{(i)}=\boldsymbol{u}^{(i)}-m_{0}^{*} \mathbf{1}$, we obtain

$$
\left\langle\left(\boldsymbol{v}^{(i)}\right)^{\prime}(s), \boldsymbol{z}\right\rangle_{\boldsymbol{V}_{0}^{*}, \boldsymbol{V}_{0}}+a\left(\boldsymbol{\xi}^{(i)}(s), \boldsymbol{z}\right)=a\left(\boldsymbol{f}^{(i)}(s), \boldsymbol{z}\right) \quad \text { for all } \boldsymbol{z} \in \boldsymbol{V}_{0},
$$

for a.a. $s \in(0, T)$. Now, $\boldsymbol{P} \boldsymbol{\xi}^{(i)}(s)=\boldsymbol{\xi}^{(i)}(s)-m\left(\boldsymbol{\xi}^{i}(s)\right) \mathbf{1} \in \boldsymbol{V}_{0}$. Therefore, we see that

$$
\begin{aligned}
a\left(\boldsymbol{\xi}^{(i)}(s), \boldsymbol{F}^{-1} \boldsymbol{z}_{0}\right) & =a\left(\boldsymbol{F}^{-1} \boldsymbol{z}_{0}, \boldsymbol{P} \boldsymbol{\xi}^{(i)}(s)\right) \\
& =\left\langle\boldsymbol{z}_{0}, \boldsymbol{P} \boldsymbol{\xi}^{(i)}(s)\right\rangle_{\boldsymbol{V}_{0}^{*}, \boldsymbol{V}_{0}} \\
& =\left(\boldsymbol{z}_{0}, \boldsymbol{P} \boldsymbol{\xi}^{(i)}(s)\right)_{\boldsymbol{H}_{0}} \\
& =\left(\boldsymbol{z}_{0}, \boldsymbol{\xi}^{(i)}(s)\right)_{\boldsymbol{H}} \text { for all } \boldsymbol{z}_{0} \in \boldsymbol{H}_{0} \subset \boldsymbol{V}_{0}^{*} .
\end{aligned}
$$


Thus, we take the difference between equation (5.37) when $i=1$ and when $i=2$. Then, we set $\boldsymbol{z}:=\boldsymbol{F}^{-1}\left(\boldsymbol{v}^{(1)}(s)-\boldsymbol{v}^{(2)}(s)\right) \in \boldsymbol{V}_{0}$, and use (2.2) to obtain

$$
\begin{aligned}
& \frac{1}{2} \frac{d}{d s}\left|\boldsymbol{v}^{(1)}(s)-\boldsymbol{v}^{(2)}(s)\right|_{\boldsymbol{V}_{0}^{*}}^{2}+\left(\boldsymbol{v}^{(1)}(s)-\boldsymbol{v}^{(2)}(s), \boldsymbol{\xi}^{(1)}(s)-\boldsymbol{\xi}^{(2)}(s)\right)_{\boldsymbol{H}} \\
& \leq\left\langle\boldsymbol{v}^{(1)}(s)-\boldsymbol{v}^{(2)}(s), \boldsymbol{f}^{(1)}(s)-\boldsymbol{f}^{(2)}(s)\right\rangle_{\boldsymbol{V}_{0}^{*}, \boldsymbol{V}_{0}} \\
& \leq \frac{1}{2}\left|\boldsymbol{v}^{(1)}(s)-\boldsymbol{v}^{(2)}(s)\right|_{\boldsymbol{V}_{0}^{*}}^{2}+\frac{1}{2}\left|\boldsymbol{f}^{(1)}(s)-\boldsymbol{f}^{(2)}(s)\right|_{\boldsymbol{V}_{0}}^{2}
\end{aligned}
$$

for a.a. $s \in(0, T)$. Now, in the variational formulation (2.16) used to construct $\boldsymbol{f}$, taking $z:=f$, we obtain

$$
|\boldsymbol{f}|_{\boldsymbol{V}_{0}}^{2} \leq \frac{1}{2 c_{p}}|\boldsymbol{g}|_{\boldsymbol{H}_{0}}^{2}+\frac{c_{p}}{2}|\boldsymbol{f}|_{\boldsymbol{H}_{0}}^{2}
$$

a.e. in $(0, T)$. Therefore, by using the Young and Poincare (3.17) inequalities,

$$
\begin{aligned}
& \frac{1}{2}\left|\boldsymbol{f}^{(1)}(s)-\boldsymbol{f}^{(2)}(s)\right|_{\boldsymbol{V}_{0}}^{2} \\
& \leq \frac{1}{2}\left|\boldsymbol{f}^{(1)}(s)-\boldsymbol{f}^{(2)}(s)\right|_{\boldsymbol{V}}^{2} \\
& \leq \frac{1}{2}\left|\boldsymbol{f}^{(1)}(s)-\boldsymbol{f}^{(2)}(s)\right|_{\boldsymbol{V}}^{2}+\frac{1}{2}\left\{\left|\boldsymbol{f}^{(1)}(s)-\boldsymbol{f}^{(2)}(s)\right|_{\boldsymbol{V}}^{2}-\left|\boldsymbol{f}^{(1)}(s)-\boldsymbol{f}^{(2)}(s)\right|_{\boldsymbol{H}_{0}}^{2}\right\} \\
& \leq \frac{1}{c_{p}}\left|\boldsymbol{f}^{(1)}(s)-\boldsymbol{f}^{(2)}(s)\right|_{\boldsymbol{V}_{0}}^{2}-\frac{1}{2}\left|\boldsymbol{f}^{(1)}(s)-\boldsymbol{f}^{(2)}(s)\right|_{\boldsymbol{H}_{0}}^{2} \\
& \leq \frac{1}{2 c_{p}^{2}}\left|\boldsymbol{g}^{(1)}(s)-\boldsymbol{g}^{(2)}(s)\right|_{\boldsymbol{H}_{0}}^{2}
\end{aligned}
$$

for a.a. $s \in(0, T)$. Then, from the monotonicity of $\beta$, we can apply the Gronwall inequality to deduce (2.8) for some positive constant $C$ that depends only on $c_{p}$ and $T$. Here, we must take care to ensure that $\boldsymbol{v}^{(1)}-\boldsymbol{v}^{(2)}=\boldsymbol{u}^{(1)}-\boldsymbol{u}^{(2)}$. If $\beta$ is a Lipschitz continuous function with Lipschitz constant $c_{\beta}>0$, then $\boldsymbol{\xi}=\left(\beta(u), \beta\left(u_{\Gamma}\right)\right)$ and, with the help of the monotonicity of $\beta$, we have

$$
\left|\boldsymbol{\xi}^{(1)}(s)-\boldsymbol{\xi}^{(2)}(s)\right|_{\boldsymbol{H}}^{2} \leq c_{\beta}\left(\boldsymbol{u}^{(1)}(s)-\boldsymbol{u}^{(2)}(s), \boldsymbol{\xi}^{(1)}(s)-\boldsymbol{\xi}^{(2)}(s)\right)_{\boldsymbol{H}} .
$$

Thus, combining (5.39), (5.40), (5.41), and (2.8), and integrating the resultant quantity over $(0, t)$ with respect to $s$, we obtain

$$
\begin{aligned}
& \left|\boldsymbol{u}^{(1)}(t)-\boldsymbol{u}^{(2)}(t)\right|_{\boldsymbol{V}_{0}^{*}}^{2}+\frac{2}{c_{\beta}} \int_{0}^{t}\left|\boldsymbol{\xi}^{(1)}(s)-\boldsymbol{\xi}^{(2)}(s)\right|_{\boldsymbol{H}}^{2} d s \\
& \leq\left|\boldsymbol{u}_{0}^{(1)}-\boldsymbol{v}_{0}^{(2)}\right|_{\boldsymbol{V}_{0}^{*}}^{2}+\int_{0}^{t}\left|\boldsymbol{u}^{(1)}(s)-\boldsymbol{u}^{(2)}(s)\right|_{\boldsymbol{V}_{0}^{*}}^{2} d s+\frac{1}{c_{p}^{2}} \int_{0}^{t}\left|\boldsymbol{g}^{(1)}(s)-\boldsymbol{g}^{(2)}(s)\right|_{\boldsymbol{H}_{0}}^{2} d s \\
& \leq C(1+T)\left\{\left|\boldsymbol{u}_{0}^{(1)}-\boldsymbol{u}_{0}^{(2)}\right|_{\boldsymbol{V}_{0}^{*}}^{2}+\int_{0}^{T}\left|\boldsymbol{g}^{(1)}(s)-\boldsymbol{g}^{(2)}(s)\right|_{\boldsymbol{H}_{0}}^{2} d s\right\}
\end{aligned}
$$

for all $t \in[0, T]$. That is, we have obtained (2.9)). 


\section{Acknowledgments}

The author wishes to express his heartfelt gratitude to Professors Goro Akagi and Ulisse Stefanelli, who kindly gave him the opportunity of exchange visits supported by the JSPSCNR bilateral joint research project on Innovative Variational Methods for Evolution Equations. The author is also indebted to Professor Pierluigi Colli, who kindly gave him the opportunity for fruitful discussions that aided in obtaining the results of this paper. The author was supported by JSPS KAKENHI Grant-in-Aid for Scientific Research(C), Grant Number 26400164.

\section{References}

[1] T. Aiki, Multi-dimensional Stefan problems with dynamic boundary conditions, Appl. Anal., 56 (1995), 71-94.

[2] T. Aiki, Periodic stability of solutions to some degenerate parabolic equations with dynamic boundary conditions, J. Math. Soc. Japan, 48 (1996), 37-59.

[3] F. Andreu, J. M. Mazón, J. Toledo and N. Igbida, A degenerate elliptic-parabolic problem with nonlinear dynamical boundary conditions, Interfaces Free Bound., 8 (2006), 447-479.

[4] V. Barbu, Nonlinear differential equations of monotone types in Banach spaces, Springer, London 2010.

[5] H. Brézis, Opérateurs maximaux monotones et semi-groupes de contractions dans les especes de Hilbert, North-Holland, Amsterdam, 1973.

[6] J. W. Cahn and J. E. Hilliard, Free energy of a nonuniform system I. Interfacial free energy, J. Chem. Phys., 2 (1958), 258-267.

[7] L. Calatroni and P. Colli, Global solution to the Allen-Cahn equation with singular potentials and dynamic boundary conditions, Nonlinear Anal., 79 (2013), 12-27.

[8] L. Cherfils, S. Gatti and A. Miranville, A variational approach to a Cahn-Hilliard model in a domain with nonpermeable walls, J. Math. Sci. (N.Y.), 189 (2013), 604-636.

[9] L. Cherfils and M. Petcu, A numerical analysis of the Cahn-Hilliard equation with non-permeable walls, Numer. Math., 128 (2014), 518-549.

[10] P. Colli and T. Fukao, Cahn-Hilliard equation with dynamic boundary conditions and mass constraint on the boundary, J. Math. Anal. Appl., 429 (2015), 1190-1213.

[11] P. Colli and T. Fukao, Equation and dynamic boundary condition of Cahn-Hilliard type with singular potentials, preprint arXiv:1502.05159 [math.AP] (2015), pp. 1-26.

[12] P. Colli and T. Fukao, Nonlinear diffusion equations as asymptotic limit of CahnHilliard system, in preparation. 
[13] P. Colli, G. Gilardi and J. Sprekels, On the Cahn-Hilliard equation with dynamic boundary conditions and a dominating boundary potential, J. Math. Anal. Appl., 419 (2014), 972-994.

[14] P. Colli and A. Visintin, On a class of doubly nonlinear evolution equations, Comm. Partial Differential Equations, 15 (1990), 737-756.

[15] A. Damlamian, Some results on the multi-phase Stefan problem, Comm. Partial Differential Equations, 2 (1977), 1017-1044.

[16] C. M. Elliott and S. Zheng, On the Cahn-Hilliard equation, Arch. Rational Mech. Anal., 96 (1986), 339-357.

[17] A. Friedman, The Stefan problem in several space variables, Trans. Amer. Math. Soc., 133 (1968), 51-87.

[18] G. Gilardi, A. Miranville and G. Schimperna, On the Cahn-Hilliard equation with irregular potentials and dynamic boundary conditions, Commun. Pure. Appl. Anal., 8 (2009), 881-912.

[19] G. R. Goldstein, A. Miranville and G. Schimperna, A Cahn-Hilliard model in a domain with non-permeable walls, Phys. D, 240 (2011), 754-766.

[20] A. Grigor'yan, Heat kernel and analysis on manifolds, American Mathematical Society, International Press, Boston, 2009.

[21] S. L. Kamenomostskaja, On Stefan's problem, Mat. Sb. (N.S.), 53 (1961), 489-514.

[22] N. Kenmochi, Monotonicity and compactness methods for nonlinear variational inequalities, in Handbook of differential equations: stationary partial differential equations. Vol. IV, M. Chipot (Ed.), North-Holland, Amsterdam (2007), 203-298.

[23] N. Kenmochi and M. Niezgódka, Viscosity approach to modelling non-isothermal diffusive phase separation, Jpn. J. Ind. Appl. Math., 13 (1996), 135-169.

[24] M. Kubo, The Cahn-Hilliard equation with time-dependent constraint, Nonlinear Anal., 75 (2012), 5672-5685.

[25] E. Magenes, C. Verdi and A. Visintin, Semigroup approach to the Stefan problem with nonlinear flux, Atti Accad. Naz. Lincei Rend. Cl. Sci. Fis. Mat. Natur. (8), 75 (1983), 24-33.

[26] J. Simon, Compact sets in the spaces $L^{p}(0, T ; B)$, Ann. Mat. Pura. Appl. (4), 146 (1987), 65-96.

[27] A. Visintin, Models of phase transitions, Birkhäuser, Boston, 1996. 